\title{
Internet Addiction: A Systematic Review of Epidemiological Research for the Last Decade
}

\author{
D. J. Kuss ${ }^{*}, 1$, M. D. Griffiths ${ }^{1}$, L. Karila ${ }^{2}$ and J. Billieux ${ }^{3}$ \\ ${ }^{1}$ International Gaming Research Unit, Nottingham Trent University, Burton Street, Nottingham NG1 4BU, UK; ${ }^{2}$ Addiction Research \\ and Treatment Center, Paul Brousse Hospital, Paris Sud-11 University, AP-HP, INSERM-CEA U1000, Villejuif, France; ${ }^{3}$ Laboratory \\ for Experimental Psychopathology, Psychological Science Research Institute, Université catholique de Louvain, Louvain-La-Neuve, \\ Belgium
}

\begin{abstract}
In the last decade, Internet usage has grown tremendously on a global scale. The increasing popularity and frequency of Internet use has led to an increasing number of reports highlighting the potential negative consequences of overuse. Over the last decade, research into Internet addiction has proliferated. This paper reviews the existing 68 epidemiological studies of Internet addiction that (i) contain quantitative empirical data, (ii) have been published after 2000, (iii) include an analysis relating to Internet addiction, (iv) include a minimum of 1000 participants, and (v) provide a full-text article published in English using the database Web of Science. Assessment tools and conceptualisations, prevalence, and associated factors in adolescents and adults are scrutinised. The results reveal the following. First, no gold standard of Internet addiction classification exists as 21 different assessment instruments have been identified. They adopt official criteria for substance use disorders or pathological gambling, no or few criteria relevant for an addiction diagnosis, time spent online, or resulting problems. Second, reported prevalence rates differ as a consequence of different assessment tools and cut-offs, ranging from $0.8 \%$ in Italy to $26.7 \%$ in Hong Kong. Third, Internet addiction is associated with a number of sociodemographic, Internet use, and psychosocial factors, as well as comorbid symptoms and disorder in adolescents and adults. The results indicate that a number of core symptoms (i.e., compulsive use, negative outcomes and salience) appear relevant for diagnosis, which assimilates Internet addiction and other addictive disorders and also differentiates them, implying a conceptualisation as syndrome with similar etiology and components, but different expressions of addictions. Limitations include the exclusion of studies with smaller sample sizes and studies focusing on specific online behaviours. Conclusively, there is a need for nosological precision so that ultimately those in need can be helped by translating the scientific evidence established in the context of Internet addiction into actual clinical practice.
\end{abstract}

Keywords: Internet addiction, literature review, epidemiology, empirical research, last decade, quantitative, large-scale.

\section{INTRODUCTION}

In contemporary society approximately $40 \%$ of the world population is online. Furthermore, global Internet usage has grown nearly six-fold over the last decade, with $96 \%$ of Internet users in Korea using high-speed Internet connections in comparison to $78 \%$ in the UK, and 56\% in the USA [1,2]. Compared to Internet access in 2000, the USA has more than doubled its usage, while mobile Internet use has increased substantially up to 2011 [3], indicating that Internet use via different hardware has become a highly prevalent activity for both adolescents and adults. From a global perspective, Google is the most popular online destination, closely followed by the social networking site Facebook ${ }^{1}$ [3]. In 2012, children and adolescents in Australia spent an average of 24 hours online per month, compared with 65 hours for those aged 18-24 years, and more than 100 hours per month in 25-34 year olds [4]. This suggests that young adults are the most active Internet users as they spend approximately three hours online per day.

The increasing popularity and frequency of Internet use has led to the emergence of clinical cases presenting abuse symptoms. Since the 1980s, school counsellors were advised to take excessive use of video games seriously as it could result in "addiction" [5]. In 1996, the concept of Internet Addiction Disorder emerged for the first time, initially as a satirical hoax as a response to the perceived pathologising of everyday behaviours [6]. Goldberg understood the condition as an analogue to substance dependence, as based on

*Address correspondence to this author at the Doctoral Researcher, International Gaming Research Unit. Nottingham Trent University, NG1 4BU Nottingham, UK; Tel: + 4478911194 90;

E-mail: daria.kuss@ntu.ac.uk

\footnotetext{
${ }^{1}$ Only in Japan, Google and Facebook fall a few places behind services such as Yahoo! 3. The Nielsen Company. State of the media: U.S. digital consumer report. The Nielsen Company2012..
}

criteria in the Diagnostic and Statistical Manual for Mental Disorders (DSM-IV) [7]. Based on this, the individual had to experience a minimum of three of the following symptoms over the period of twelve months: tolerance, withdrawal, lack of control, relapse, large amounts of time spent online, negative consequences, and continuation of use irrespective of problem awareness [6]. Following this initial proposal, Griffiths and Young [8,9] emerged as the pioneers of early research into Internet addiction as they were the first to scrutinise the phenomenon empirically. Modelling the Internet addiction criteria after the APA's substance dependence diagnosis [7], Young [10] presented the case of a female homemaker who progressively increased her engagement in chat rooms because of her growing commitment to virtual communities, which have been described as offering emotional support and a platform for discussion and information [11]. The homemaker spent increasing amounts of time online to the detriment of her real life responsibilities and eventually developed withdrawal symptoms [10]. This case exemplified for the first time that the stereotypical view of the excessive Internet user, i.e., a young male technophile, had to be overthrown and in its place appeared a female user seeking a sense of belonging and comfort on the Internet. Griffiths [12] also published case study accounts including both males and females. Following these initial case reports, Young [13] was among the first to present findings from an exploratory survey comprising 396 dependent Internet users who endorsed a minimum of five out of eight criteria adapted from a diagnosis of pathological gambling [7], and 100 non-dependent Internet users. On average, the dependent users spent eight times more hours online than the controls, and used chat rooms and $\mathrm{MUDs}^{2}$ more frequently [13].

\footnotetext{
2 Multi-User Dungeons, the exclusively textual precursors of today's Massively Multiplayer Online Role-Playing Games (MMORPGs).

*Address correspondence to this author at the Doctoral Researcher, International Gaming Research Unit. Nottingham Trent University, NG1 4BU Nottingham, UK; Tel: + 4478911194 90; E-mail: daria.kuss@ntu.ac.uk
} 
These early studies can be seen as the beginning of empirical research into the area of Internet addiction.

Since these initial efforts to shed light upon an emerging mental health problem, empirical research into Internet addiction has greatly increased. Various terms have been used to name the condition, including compulsive computer use [14], Internet dependency [15], pathological Internet use [16], problematic Internet use [17], virtual addiction [18], and Internet addiction disorder [19]. Recently, the APA [20] published the updated version of the DSM and included Internet Gaming Disorder in the appendix as condition that requires further empirical and clinical research. In the DSM-5, Internet Gaming Disorder includes nine criteria, namely preoccupation, withdrawal, tolerance, loss of control, continued use irrespective of problem awareness, neglect of alternative recreational activities, escapism and mood modification as usage motivations, deception, and jeopardisation of relationships and job. This clearly situates the behaviour within the new diagnostic entity of Addiction and Related Disorders. Five or more symptoms need to be met over a 12-month period for diagnosis which must cause the individual clinically significant impairment or distress [20, 21]. The conflation of Internet use and online gaming in this diagnostic category creates further diagnostic imprecision as seven out of the nine criteria relate to gaming specifically. Therefore, although the inclusion of Internet Gaming Disorder in the research appendix of the DSM-5 emphasise the necessity for further research, the new research diagnosis appears somewhat crude and vague, further complicating a clinical evaluation. Although empirical research over the last decade has significantly increased, the classification of Internet addiction is still controversial as no gold standard of Internet addiction assessment has emerged. A number of review papers on Internet addiction have been published since 2005 [22-25]. Some of the most recently published reviews specifically integrated treatment outcome research [26-28] and comorbidity [29], while others have looked at the biological basis and the psychological factors involved in the aetiology for the disorder [e.g., 30, 31]. Another study [32] suggests that current Internet addiction assessment tools tap into the following dimensions of addiction: compulsive use, negative outcomes, salience, withdrawal symptoms, mood regulation, escapism and social comfort, which are comparable with Griffiths' [33] behavioural addiction components. These reviews highlight the dissimilarity in assessment across studies that impede the possibility of cross-comparisons as well as an evaluation of the epidemiological prevalence rates across samples. In order to elucidate the potential problem of Internet addiction, the aim of this paper is to review the epidemiological Internet addiction research of the last decade. This review sets out to answer the following research questions: (i) what is Internet addiction (i.e., how is it assessed)?, (ii) how common is it?, and (iii) what are the associated factors?

\section{METHOD}

A literature search was conducted using the database Web of Science. This database was used as it is more comprehensive than other commonly used databases, such as Psycinfo or PubMed because it includes various multidisciplinary databases. The following search terms (and their derivatives) were entered with regards to Internet addiction specifically: 'Internet' or 'online' and 'excessive', 'problematic', 'compulsive', and 'addictive'. Studies were selected based on the following inclusion criteria. Studies had to (i) contain quantitative empirical data, (ii) have been published after 2000, (iii) include an analysis relating to Internet addiction, (iv) include a minimum of 1000 participants, and (v) provide a fulltext article published in English. For comparison purposes, studies focusing solely on particular online applications (e.g., gaming, social networking) were excluded from analysis. The databases were searched in April and May 2013. The initial search yielded 1,332 results. Following a thorough inspection of the articles' titles and abstracts, the articles not meeting the inclusion criteria were excluded. Data were organised with regards to assessment approach, prevalence, and factors associated with Internet addiction.

\section{RESULTS}

A total of 69 epidemiological research papers were identified from the literature search that met the initial inclusion criteria. However, one study [34] had to be excluded as it did not provide sufficient information on how Internet addiction was assessed. Therefore, a total of 68 studies were included in this literature review. The first part of the results section will present the assessment approaches adopted, as they highlight the various conceptualisations of Internet addiction, which will be classed in accordance with the specific samples used, namely adolescents and adults. Three main diagnostic assessment approaches comprised Young's Internet Addiction Test and Internet Addiction Diagnostic Questionnaire [13, 35], Chen et al.'s Chinese Internet Addiction Scale [36], and various miscellaneous approaches for classification. The next part will summarize the reported prevalence rates, which will be followed by the last part that outlines the factors that that have been found to be statistically associated with Internet addiction.

\subsection{What is Internet addiction? Assessment tools and Conceptualisations}

\subsubsection{The Internet Addiction Test and the Internet Addiction Diagnostic Questionnaire}

Two related, but slightly different tools for Internet addiction assessment have been developed by Young $[13,35]$. The Internet Addiction Test (IAT) [35] is a 20-item self-report scale that assesses Internet addiction as based on criteria for substance dependence and pathological gambling [7]. The criteria include loss of control, neglecting everyday life, relationships and alternative recreation activities, behavioural and cognitive salience, negative consequences, escapism/mood modification, and deception, and are rated on a Likert scale ranging from 1 ("not at all") to 5 ("always"), allowing a dimensional rather than categorical assessment. Internet users are classed as having significant problems due to Internet use if they score 70-100, and having frequent problems when scoring 40-69 [35]. The internal consistency of the IAT has been reported as satisfactory, with a Cronbach's alpha of .84 [37]. The IAT does not contain a temporal dimension by asking the participant to rate the presence of the symptoms over a specified period of time. Moreover, the cut-offs appear rather arbitrary as they are not based on empirical considerations, such as a clinical evaluation of disorder severity based on the presence and impact of symptoms. A recent study [38] including a Greek adolescent sample indicates that a lower cut-off point of 51 presents the highest specificity and sensitivity. This finding raises issues concerning the cultural context of analysis, suggesting that sociocultural factors impact upon Internet addiction assessment.

The Internet Addiction Diagnostic Questionnaire (IADQ) [13] is a parsimonious 8-item self-report measure based on the diagnostic symptoms of pathological gambling [7]. The criteria utilised for the IADQ include preoccupation, tolerance, loss of control, withdrawal, negative consequences, denial, and escapism. Two of the original ten criteria for pathological gambling (i.e., committing illegal acts to finance the behaviour and reliance on others for money) were omitted to produce a "slightly more rigorous cut-off score" [13]. Endorsing five or more of the criteria indicates Internet addiction.

\subsubsection{Chen's Internet Addiction Scale}

Chen's Internet Addiction Scale (CIAS) [36] was the most frequently used scale in the included empirical research papers as a total of 16 studies made use of it to assess Internet addiction. The CIAS is a 26-item self-report measurement scored on a 4-point 
Likert scale, assessing the core symptoms of Internet addiction, tolerance, compulsive use, and withdrawal, as well as related problems in terms of negative impact on social activities, interpersonal relationships, physical condition, and time management. In addition to this, it inquires into weekly online hours and Internet use experience. The internal consistency of the scale was found to be good, with Cronbach's alpha values between .79 to .93 for the respective subscales [36]. It has also been reported that the screening cut-off of $57 / 58$ points has high sensitivity, and the diagnostic cut-off point of $63 / 64$ as performed by psychiatrists revealed the highest diagnostic accuracy with $87.6 \%$ of patients diagnosed with Internet addiction appropriately [39]. Adopted cutoff points for Internet addiction varied marginally between studies, as scores of 63/64 or 67/68 have been used as cut-offs for Internet addiction classification, without the respective authors specifying reasons for their choice, such as the instrument's factor structure.

\subsubsection{Miscellaneous Diagnostic Assessment Tools}

The remaining assessment tools represent a plethora of newly designed measurement instruments or alternative criteria based on which Internet addiction and Internet use-related problems have been categorised. A total of 21 studies were identified that used miscellaneous criteria. Of these, 14 studies used miscellaneous criteria to identify Internet addiction in adolescents [40-52]. In addition to the adolescent samples, miscellaneous classification criteria for Internet addiction have been used in adult samples, including a total of eight studies [53-60]. Classifications vary tremendously, ranging from the adoption of official criteria for substance use disorders or pathological gambling, to no or few criteria relevant for an addiction diagnosis. In yet other cases excessive use is assessed based on how much time is spent online or how many problems occur as a consequence of use, providing an overly simplistic picture of Internet addiction. Detailed information concerning each of the assessment instruments, criteria, and problems with the respective classifications are provided in Table $\mathbf{1}$.

\subsection{How Common is Internet Addiction?}

\subsubsection{Prevalence of Internet Addiction in Adolescents}

A total of seven studies used the IAT for Internet addiction assessment in adolescents and children aged 8 to 24 years [61-67], with sample sizes ranging from 1,618 [65] to 17,599 participants [63]. Although the same measurement instrument has been used in these studies, various cut-offs have been applied to demarcate addiction or excessive use across studies. Reported prevalence rates varied significantly with $0.8 \%$ in Italian high school students were considered to be seriously addicted [62], and $20.3 \%$ of adolescents and $13.8 \%$ of children in a South Korean sample were classed as addicted to using the Internet [67].

In eleven studies, the IADQ [13] was used to assess Internet addiction in adolescents [68-78]. The sample sizes ranged from 1,270 in Greece $[71,72]$ to 10,988 adolescents and young adults in China, aged 13-23 years [78] The same cut-off, i.e., endorsing a minimum of five out of eight diagnostic items, has been applied to a majority of these studies. Internet addiction prevalence rates ranged from $1.7 \%$ of boys and $1.4 \%$ of girls in a representative sample of Finnish adolescents [77] to $26.4 \%$ and $26.7 \%$ at wave one and wave two in a longitudinal sample of adolescent students in Hong Kong, respectively [73]. The reported prevalence rates in Asian adolescents have been found to be significantly higher in comparison to both, Western countries, as well as samples of children.

Chen's Internet Addiction Scale was used in nine studies including adolescent samples [79-87]. The sample sizes ranged from 1,890 students in Taiwan [87] to including 9,405 in Southern Taiwan [80]. In all of these studies, the relatively liberal cut-off point of $63 / 64$ on the CIAS has been applied. Prevalence estimates varied substantially, with the lowest rate of $10.8 \%$ found in an adolescent sample in Southern Taiwan [79], whereas in other adolescents samples in Southern Taiwan prevalence rates between $18 \%$ and $21 \%$ were reported [80, 82-87].

A total of 13 studies used miscellaneous criteria to identify Internet addiction in adolescents [40-52]. Sample sizes varied from including 1,098 adolescents in Singapore [43] to 73,238 adolescents in South Korea [51]. Sung and colleagues [51] used the Internet Addiction Proneness Scale - Short Form (KS-Scale) [88] in a sample of 73,238 adolescents in South Korea and reported that $3.0 \%$ and $11.9 \%$ of adolescents were at high risk and at potential risk for developing Internet addiction in South Korea, respectively [51]. On the other end of the spectrum, Xu et al. [45] used the DRM 52 Scale of Internet use in a random sample of 5,122 adolescents in Shanghai, China with the result that $8.8 \%$ of adolescents in this sample were classified as Internet addicts. The only cross-cultural study of Internet addiction prevalence included two separate samples of 1,761 high school students in China and 1,182 students in the USA who were used in a longitudinal study by Sun et al. [47] using the (CIUS), and showed that the prevalence rates were $5.8 \%$ in Chinese females, $15.7 \%$ in Chinese males, $9.7 \%$ in US females, and $7.3 \%$ in US males. A detailed summary of the epidemiological studies that assessed Internet addiction prevalence in adolescents is provided in Table 2 .

\subsubsection{The Prevalence of Internet Addiction in Adults}

In six studies, Young's Internet Addiction Test [35] was used to assess Internet addiction in adults [89-94]. The sample sizes ranged from 1,034 young adults in Turkey [90] to 13,588 Internet users in Korea [93]. Similar to the usage of the IAT in adolescent samples, in the adult samples, various cut-off criteria have been utilised in order to demarcate Internet addiction from non-pathological Internet usage behaviours. Reported prevalence rates using the IAT ranged from $1.2 \%$ of Internet users in the UK [92] to $9.7 \%$ of Turkish college students [90].

The Internet Addiction Diagnostic Questionnaire [13] was used in three adult samples [95-97]. The reported Internet addiction prevalence rates in these studies were notably diverse as in a sample of Norwegian adults, $1.0 \%$ [96] and in a sample of 1,856 Iranian Internet users $22.8 \%$ [95] were found to be addicted to the Internet.

Chen's Internet Addiction Scale was used in seven studies including adult samples [98-104]. All samples included college or university students in Taiwan. Sample sizes ranged from 1,360 university freshmen [100] to 4,456 college students [103]. The studies that reported prevalence rates used teenage samples. Using the rather conservative cut-off of $67 / 68$ on the CIAS, relatively similar prevalence rates of $12.9 \%$ and $12.3 \%$ have been reported by Yen et al. in Taiwan $[101,102]$, ranging up to $17.9 \%$ as reported by Tsai et al. [100].

Miscellaneous classification criteria for Internet addiction have been used in a total of eight studies including adult samples [5360]. All sample sizes were between 1000 and 2000 participants, with the exception of a sample of 16,925 regular Internet users in the Netherlands [53]. Prevalence rates varied, ranging from $1.8 \%$ of a sample of 1,147 participants in Sweden (age range 15-94 years) who experienced all of the inquired problems due to Internet use [58], whereas Demetrovics and colleagues [55] reported that of a sample of 1,037 Hungarian young adults, 4.3\% had significant problems because of their Internet use as measured via the PIUQ. A complete summary of the epidemiological studies of Internet addiction in adults is provided in Table $\mathbf{3}$.

\subsection{What are the Associated Factors?}

Four main factors have been found to be associated with Internet addiction. A visual representation of these factors is presented in (Fig. 1). 
Table 1. Internet Addiction Assessment Instruments.

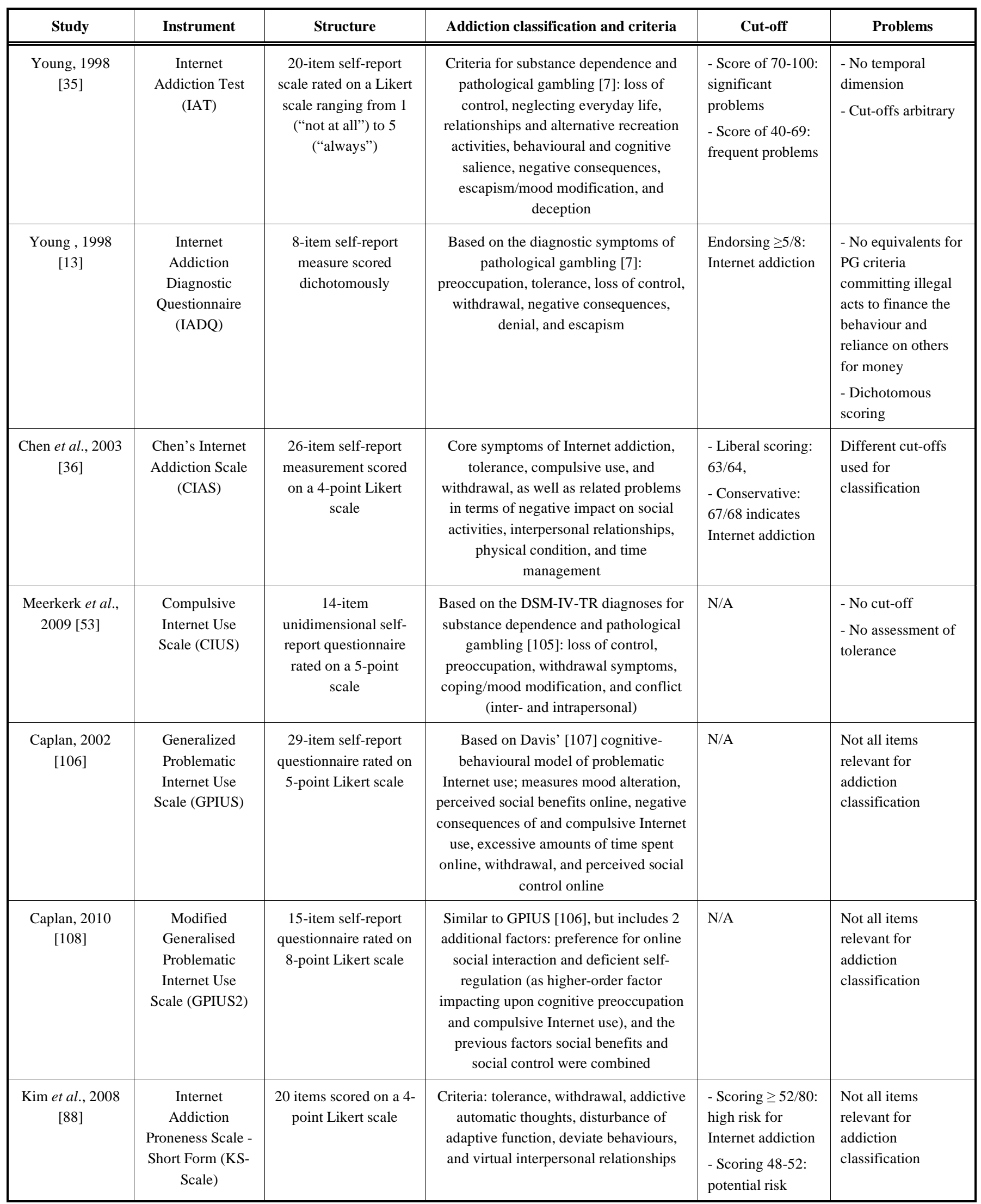


(Table 1) Contd....

\begin{tabular}{|c|c|c|c|c|c|}
\hline Study & Instrument & Structure & Addiction classification and criteria & Cut-off & Problems \\
\hline $\begin{array}{c}\text { Sun et al., } 2012 \\
{[47]}\end{array}$ & $\begin{array}{l}\text { Compulsive } \\
\text { Internet Use } \\
\text { Scale (CIUS) }\end{array}$ & $\begin{array}{l}4 \text { items on 5-point } \\
\text { Likert scale }\end{array}$ & $\begin{array}{c}\text { Based on Davis et al.'s [17] Online } \\
\text { Cognition Scale }\end{array}$ & $\begin{array}{l}\text { Scoring mean of } \\
\text { 4/possible } 5 \text { : } \\
\text { Internet addiction }\end{array}$ & $\begin{array}{l}\text { No use of } \\
\text { recognised } \\
\text { diagnostic criteria }\end{array}$ \\
\hline $\begin{array}{c}\text { Liu et al., } 2011 \\
{[48]}\end{array}$ & $\begin{array}{l}\text { Problematic } \\
\text { Internet Use } \\
\text { Scale (PIU) }\end{array}$ & $\begin{array}{l}6 \text { items scored } \\
\text { dichotomously }\end{array}$ & $\begin{array}{l}\text { Based on Minnesota Impulsive Disorder } \\
\text { Inventory [110] }\end{array}$ & $\begin{array}{l}\text { Endorsing } \\
\text { craving, } \\
\text { withdrawal, } \\
\text { abstinence } \\
\text { attempts } \\
\text { simultaneously: } \\
\text { problematic } \\
\text { Internet use }\end{array}$ & $\begin{array}{l}\text { Overly simplistic } \\
\text { classification }\end{array}$ \\
\hline $\begin{array}{l}\text { Mythily et al., } \\
2008 \text { [50] }\end{array}$ & $\begin{array}{l}\text { Excessive } \\
\text { Internet use }\end{array}$ & $\begin{array}{l}\text { Daily hours spent } \\
\text { online }\end{array}$ & Length of daily Internet use & $\begin{array}{l}\text { Spending } \geq \\
\text { 5hours } \\
\text { online/daily: } \\
\text { excessive Internet } \\
\text { use }\end{array}$ & $\begin{array}{l}\text { Overly simplistic } \\
\text { classifcation }\end{array}$ \\
\hline $\begin{array}{l}\text { Wölfling et al., } \\
2010[111]\end{array}$ & $\begin{array}{l}\text { Assessment for } \\
\text { Computer and } \\
\text { Internet } \\
\text { Addiction- } \\
\text { Screener (AICA- } \\
\text { S) }\end{array}$ & $\begin{array}{l}16 \text { items scored on 5- } \\
\text { point Likert scale }\end{array}$ & $\begin{array}{l}\text { Based on diagnostic criteria of substance } \\
\text { dependence by DSM-IV-TR [105] and } \\
\text { ICD-10 [112]; criteria: craving, } \\
\text { tolerance, withdrawal, loss of control, } \\
\text { preoccupation and negative } \\
\text { consequences concerning poorer health, } \\
\text { family conflicts or deteriorating } \\
\text { achievements, mood modification }\end{array}$ & $\begin{array}{l}\text { Scoring } \geq \\
\text { 13.5/27: Internet } \\
\text { addiction }\end{array}$ & $\begin{array}{l}\text { Lack of time } \\
\text { criterion }\end{array}$ \\
\hline $\begin{array}{c}\text { Thatcher \& } \\
\text { Goolam, } 2005 \\
{[113]}\end{array}$ & $\begin{array}{l}\text { Problematic } \\
\text { Internet Use } \\
\text { Questionnaire } \\
\text { (PIUQ) }\end{array}$ & $\begin{array}{l}20 \text { items scored on 5- } \\
\text { point Likert scale }\end{array}$ & $\begin{array}{l}\text { Based on Young's criteria for Internet } \\
\text { addiction [10] and the South Oaks } \\
\text { Gambling Screen [114], assesses online } \\
\text { preoccupation, adverse effects, and } \\
\text { online social interactions }\end{array}$ & N/A & $\begin{array}{l}\text { Not all items } \\
\text { relevant for } \\
\text { addiction } \\
\text { classification }\end{array}$ \\
\hline
\end{tabular}


(Table 1) Contd....

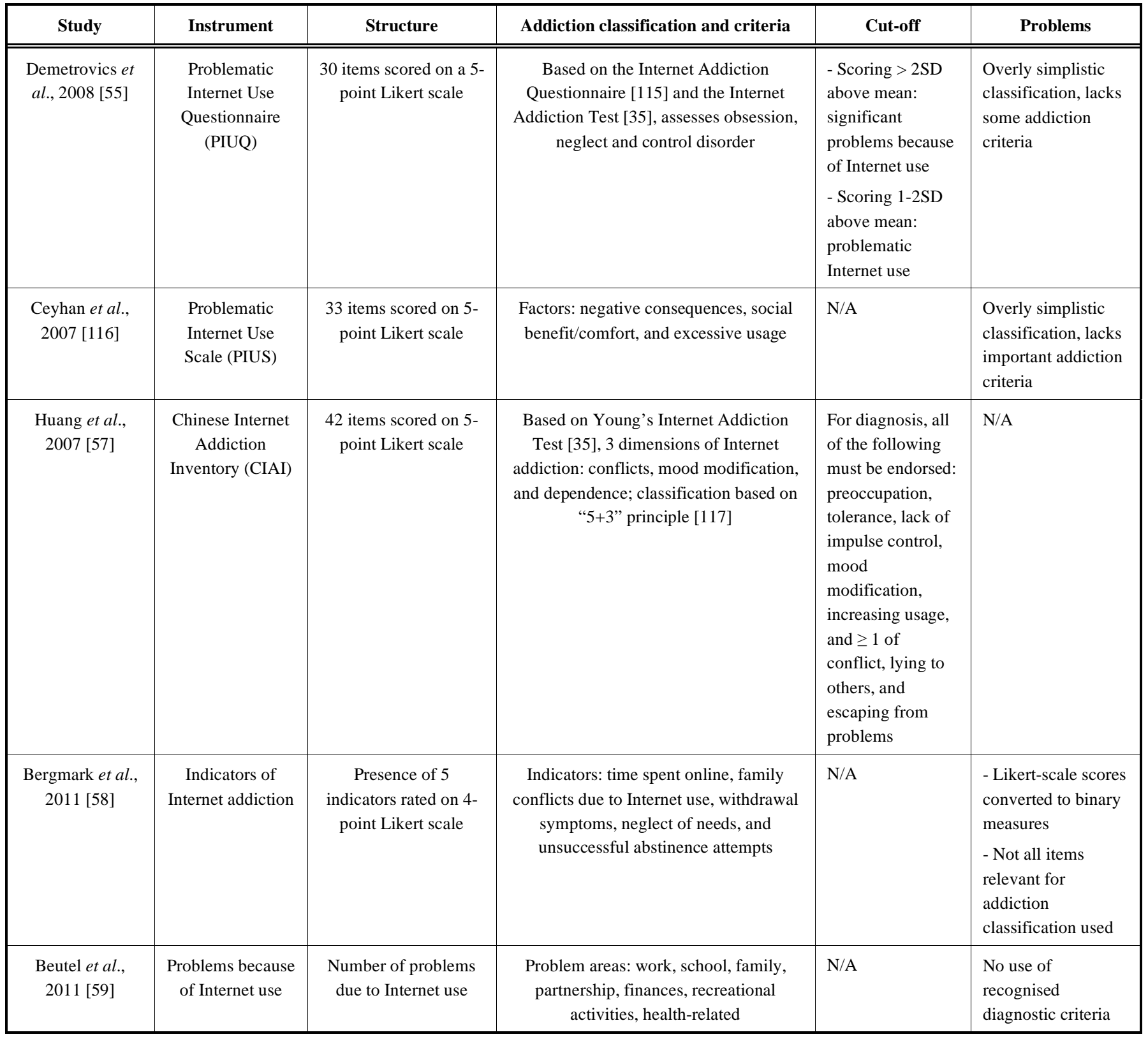

Table 2. Epidemiological Internet Addiction Studies in Adolescents.

\begin{tabular}{|c|c|c|c|c|}
\hline Study & Sample and design ${ }^{1}$ & Instruments & $\begin{array}{l}\text { Addiction classification } \\
\text { and criteria }\end{array}$ & Results \\
\hline $\begin{array}{l}\text { Ak et al., } \\
2013[61]\end{array}$ & $\begin{array}{c}\quad N=4,311 \text { adolescents in } \\
\text { Turkey (46\% male, age range } \\
15-19 \text { years) }\end{array}$ & $\begin{array}{l}\text { - Turkish version of Internet Addiction } \\
\text { Test (IAT) [13] }\end{array}$ & $\begin{array}{l}\text { - Scoring } \geq 60 / 100 \text { on the } \\
\text { IAT }=\text { excessive Internet } \\
\text { users }\end{array}$ & $\begin{array}{l}\text { - } 5 \% \text { excessive users } \\
\text { - Predictors of Internet } \\
\text { addiction: Internet access at } \\
\text { home, male gender, family } \\
\text { income }\end{array}$ \\
\hline $\begin{array}{l}\text { Poli \& } \\
\text { Agrimi, } \\
2012[62]\end{array}$ & $\begin{array}{l}N=2,533 \text { high school students } \\
\text { in Cremona, Italy }(44.3 \% \\
\text { males, mean age }=16.4 \text { years, } \\
\quad S D=1.51, \text { range } 14-21)\end{array}$ & $\begin{array}{l}\text { - Italian version of the Internet Addiction } \\
\text { Test (IAT) [13] }\end{array}$ & $\begin{array}{l}- \text { Scoring } 50-79 / 100= \\
\text { moderately addicted } \\
\text { - Scoring } \geq 80= \\
\text { seriously addicted }\end{array}$ & $\begin{array}{l}\text { - } 5.01 \% \text { moderately and } 0.79 \% \\
\text { seriously addicted to the } \\
\text { Internet } \\
\text { - Higher prevalence in males }\end{array}$ \\
\hline
\end{tabular}


(Table 2) Contd...

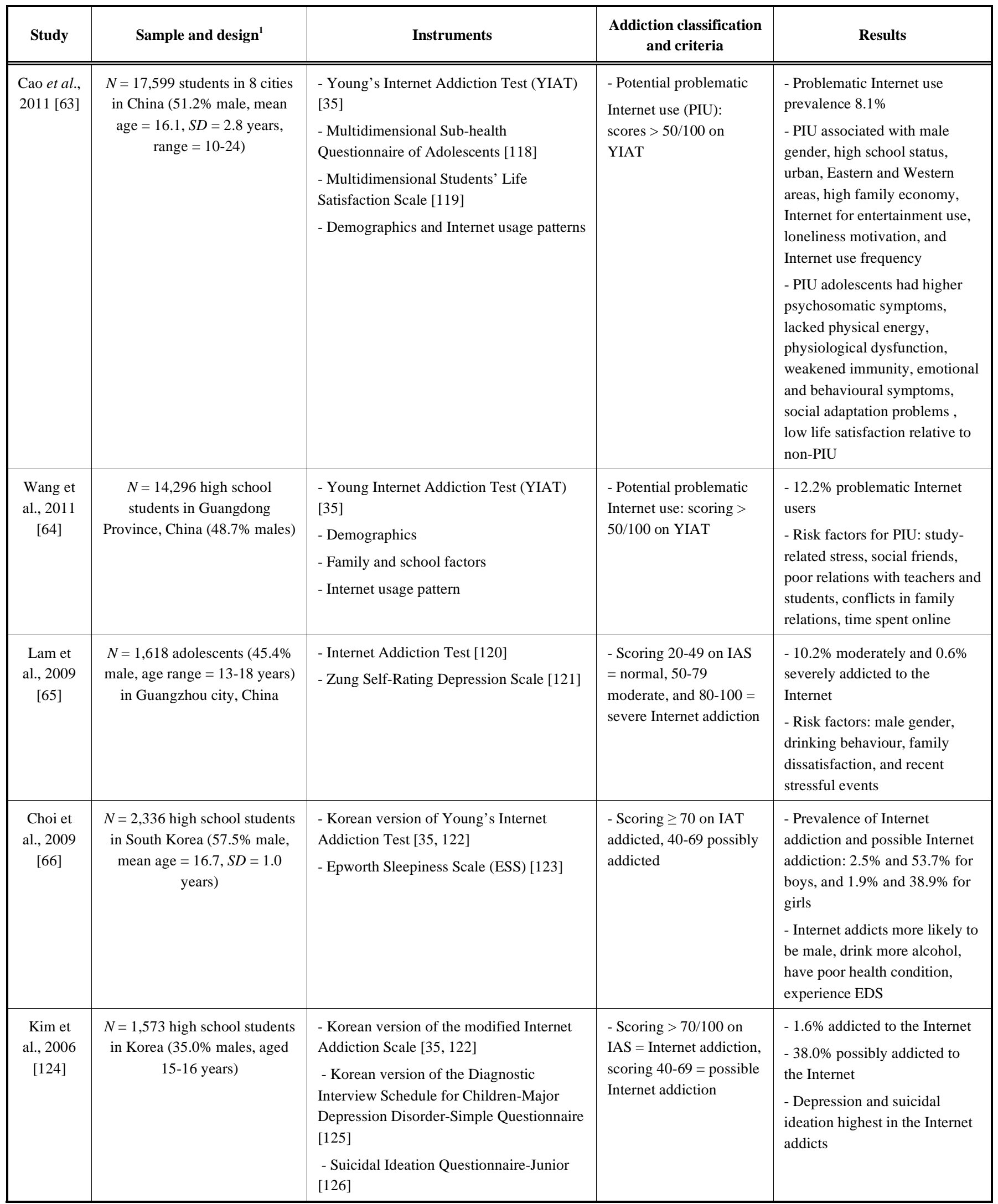


(Table 2) Contd....

\begin{tabular}{|c|c|c|c|c|}
\hline Study & Sample and design ${ }^{1}$ & Instruments & $\begin{array}{l}\text { Addiction classification } \\
\text { and criteria }\end{array}$ & Results \\
\hline $\begin{array}{l}\text { Ha et al., } \\
2006[67]\end{array}$ & $\begin{array}{c}\text { - Structured clinical interview } \\
\text { - } N \mathrm{~s}=455 \text { children }(50.3 \% ; \text { mean } \\
\text { age }=11, S D=.9 \text { years }) \text { and } 836 \\
\text { adolescents }(92.9 \% \text { male; mean } \\
\text { age }=15.8, S D=.8 \text { years }) \\
\text { - Of Internet addicts, } 12 \text { children } \\
\text { and } 12 \text { adolescents randomly } \\
\text { selected for psychiatric } \\
\text { evaluation }\end{array}$ & $\begin{array}{l}\text { - Young's Internet addiction scale } \\
\text { - K-SADS-PL-K for children } \\
\text { - SCID-IV for adolescents }\end{array}$ & - Cut- off of 80 & $\begin{array}{l}\text { - Internet addiction prevalence } \\
\text { in adolescents } 20.3 \% \text {, in } \\
\text { children } 13.8 \% \\
\text { - In child Internet addiction } \\
\text { group, } 7 \text { with ADHD } \\
\text { - In adolescent Internet } \\
\text { addiction group, } 3 \text { with } \\
\text { depression, } 1 \text { schizophrenia, } 1 \\
\text { obsessive-compulsive disorder }\end{array}$ \\
\hline $\begin{array}{l}\text { Guo et al., } \\
2012[68]\end{array}$ & $\begin{array}{c}N=3,254 \text { children (mean age }= \\
12.56, S D=1.83 \text { years; age range } \\
=8-17 \text {-years), with } \mathrm{n}=1143 \text { left } \\
\text { behind children (LBC; } 49.9 \% \\
\text { male), } \mathrm{n}=574 \text { migrant children } \\
\text { (MC; } 57.1 \% \text { male), and } \mathrm{n}=1287 \\
\text { non-left-behind rural children } \\
\text { (RC; } 51.8 \% \text { male) in China }\end{array}$ & $\begin{array}{l}\text { - Young's 8-item Internet Addiction } \\
\text { Scale [13] } \\
\text { - Children's Depression Inventory-Short } \\
\text { Form (CDI-S) [127] } \\
\text { - Nutritional status, health condition and } \\
\text { health behaviours }\end{array}$ & $\begin{array}{l}\text { - Endorsing } \geq 5 / 8 \text { items } \\
\text { on IAT }=\text { Internet } \\
\text { addicted }\end{array}$ & $\begin{array}{l}\text { - Internet addiction prevalence } \\
=3.7 \% \text { in } \mathrm{RC}, 6.4 \% \text { in } \mathrm{MC} \text { and } \\
3.2 \% \text { in } \mathrm{LBC} \\
\text { - } \mathrm{LBC} \text { and } \mathrm{MC} \text { with Internet } \\
\text { addiction, and } \mathrm{MC} \text { without } \\
\text { Internet addiction more at risk } \\
\text { for depression than RC with no } \\
\text { Internet addiction }\end{array}$ \\
\hline $\begin{array}{l}\text { Siomos et } \\
\text { al., 2012 } \\
{[69]}\end{array}$ & $\begin{array}{c}N=2,017 \text { teenage students } \\
(51.8 \% \text { males, boys' mean age }= \\
15.05, S E=.05 ; 8, \text { girls' mean age } \\
=15.08, S E=.05 ; \text { overall age } \\
\text { range }=12-19) \text { in Greece, and } n= \\
1,214 \text { parents }\end{array}$ & $\begin{array}{l}\text { - Diagnostic Questionnaire for Internet } \\
\text { Addiction (YDQ) [13] } \\
\text { - Greek version of Adolescent Computer } \\
\text { Addiction Test (ACAT; modelled after } \\
\text { Internet Addiction Test) [128] } \\
\text { - Parental Bonding Instrument [129] }\end{array}$ & $\begin{array}{l}\text { - Scoring min. } 5 / 8 \\
\text { indicates Internet } \\
\text { addiction }\end{array}$ & $\begin{array}{l}\text { - } 15.2 \% \text { addicted to the } \\
\text { Internet, } 26.9 \% \text { moderately } \\
\text { addicted } \\
\text { - Internet addiction predicted } \\
\text { by parental bonding, not } \\
\text { parental security practices } \\
\text { - Online activities associated } \\
\text { with Internet addiction: online } \\
\text { pornography, gambling, and } \\
\text { gaming }\end{array}$ \\
\hline $\begin{array}{l}\text { Fisoun et } \\
\text { al., } 2012 \\
{[72]}\end{array}$ & $\begin{array}{c}N=1,270 \text { adolescent students on } \\
\text { Kos }(48.3 \% \text { male, mean age }= \\
15.99, S E=.05, \text { girls' mean age }= \\
16.02, S E=\begin{array}{c}.05, \text { age range } 14-18 \\
\text { years })\end{array}\end{array}$ & $\begin{array}{l}\text { - Diagnostic Questionnaire for Internet } \\
\text { Addiction (YDQ) [13] } \\
\text { - Internet Addiction Test [13] } \\
\text { - Demographic questions }\end{array}$ & N/A & $\begin{array}{l}\text { - } 5.3 \% \text { addicted users, } 14.7 \% \\
\text { heavy Internet users } \\
\text { - Correlations between } \\
\text { antisocial and aggressive } \\
\text { behaviours with Internet abuse } \\
\text { regarding interest-driven } \\
\text { activities for boys, and } \\
\text { communication activities for } \\
\text { girls }\end{array}$ \\
\hline $\begin{array}{l}\text { Fisoun et } \\
\text { al., } 2012 \\
\text { [71] }\end{array}$ & $\begin{array}{c}N=1,270 \text { adolescent students on } \\
\text { Kos }(48.3 \% \text { male, mean age }= \\
15.99, S E=.05, \text { girls' mean age }= \\
16.02, S E=\begin{array}{c}.05, \text { age range } 14-18 \\
\text { years })\end{array}\end{array}$ & $\begin{array}{l}\text { - Internet Addiction Test [13] } \\
\text { - Demographic questionnaire, incl. } \\
\text { questions on substance use } \\
\text { - Eysenck's Personality Questionnaire } \\
{[130]}\end{array}$ & $\begin{array}{l}\text { - Scoring } 5 / 8 \text { on IAT }= \\
\text { addicted to the Internet }\end{array}$ & $\begin{array}{l}-7.2 \% \text { of males, and } 5.1 \% \text { of } \\
\text { females addicted to the Internet } \\
\text { - Internet and substance abusers } \\
\text { share personality } \\
\text { characteristics, i.e., } \\
\text { psychoticism } \\
\text { - Pathological Internet use } \\
\text { severity related to illicit } \\
\text { substance use }\end{array}$ \\
\hline
\end{tabular}


(Table 2) Contd....

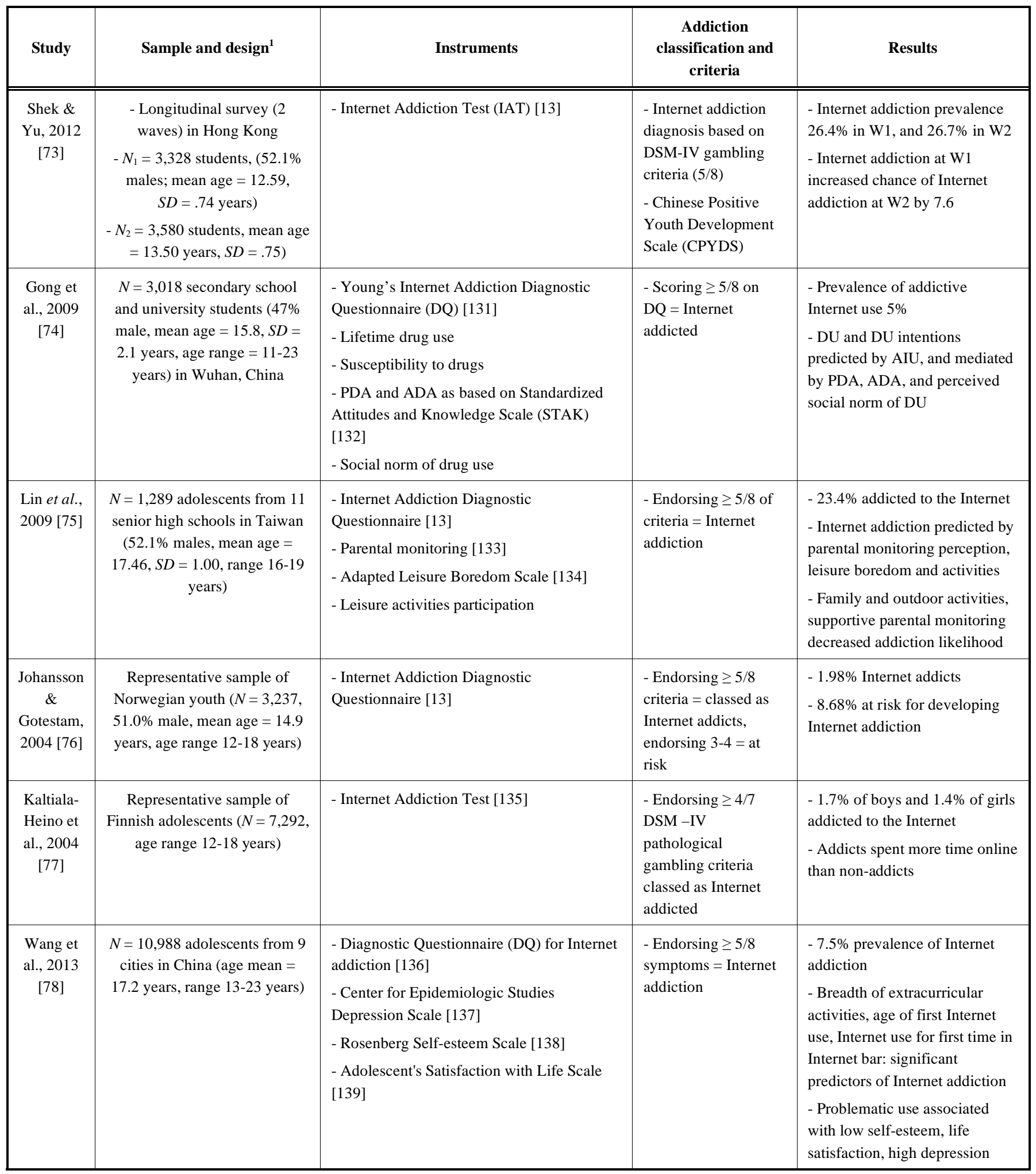


(Table 2) Contd....

\begin{tabular}{|c|c|c|c|c|}
\hline Study & Sample and design ${ }^{1}$ & Instruments & $\begin{array}{c}\text { Addiction classification and } \\
\text { criteria }\end{array}$ & Results \\
\hline $\begin{array}{l}\text { Ko et al., } \\
2009[79]\end{array}$ & $\begin{array}{l}\text { - 2-year prospective study } \\
-N=2,293 \text { adolescents } \\
(51.4 \% \text { male, mean age }= \\
12.36, S D=.55 \text { years }) \text { in } \\
\text { Southern Taiwan }\end{array}$ & $\begin{array}{l}\text { - Chen Internet Addiction Scale [36] } \\
\text { - Modified Vanderbilt ADHD Diagnostic } \\
\text { Parent Rating Scale [140] } \\
\text { - Mandarin Chinese version of the Center } \\
\text { for Epidemiological Studies Depression } \\
\text { Scale (CES-D) [137] } \\
\text { - Brief Version of the Fear of Negative } \\
\text { Evaluation Scale (BV-FNE) [141] } \\
\text { - Buss-Durkee Hostility Inventory- } \\
\text { Chinese Version-Short Form (BDHIC- } \\
\text { SF) [142] }\end{array}$ & $\begin{array}{l}\text { - Scoring } \geq 64 / 104 \text { on CIAS }= \\
\text { addicted to the Internet }\end{array}$ & $\begin{array}{l}\text { - } 10.8 \% \text { addicted to the Internet } \\
\text { - Depression, ADHD, social } \\
\text { phobia, and hostility predicted } \\
\text { Internet addiction } \\
\text { - Hostility predicted Internet } \\
\text { addiction in males and ADHD } \\
\text { in predicted Internet addiction } \\
\text { in females }\end{array}$ \\
\hline $\begin{array}{l}\text { Ko et al., } \\
2008 \text { [82] }\end{array}$ & $\begin{array}{c}N=2,114 \text { high school } \\
\text { students }(57.0 \% \text { male, } \\
\text { mean age }=16.26, S D= \\
.99 \text { years, range } 15-23 \\
\text { years) in Taiwan }\end{array}$ & $\begin{array}{l}\text { - Chen Internet Addiction Scale (CIAS) } \\
\text { [36] } \\
\text { - CRAFFT Substance Abuse Screening } \\
\text { Test [150] } \\
\text { - Behaviour inhibition system and } \\
\text { behaviour approach system Scale } \\
\text { (BIS/BAS) [151] } \\
\text { - Alcohol attitudes } \\
\text { - Rosenberg Self-Esteem Scale (RSES) } \\
\text { [145] } \\
\text { - Family APARG Index (APGAR) [144] } \\
\text { - Brief Multidimensional Students' Life } \\
\text { Satisfaction Scale (BMSLSS) [152] }\end{array}$ & $\begin{array}{l}\text { - Scoring } \geq 63 / 104 \text { on CIAS } \\
\text { indicated Internet addiction }\end{array}$ & $\begin{array}{l}\text { - Prevalence of Internet } \\
\text { addiction } 18.3 \% \\
\text { - Internet addiction associated } \\
\text { with problematic alcohol use } \\
\text { and psychosocial variables (i.e., } \\
\text { psychosocial proneness for } \\
\text { problem behaviours) }\end{array}$ \\
\hline
\end{tabular}


(Table 2) Contd....

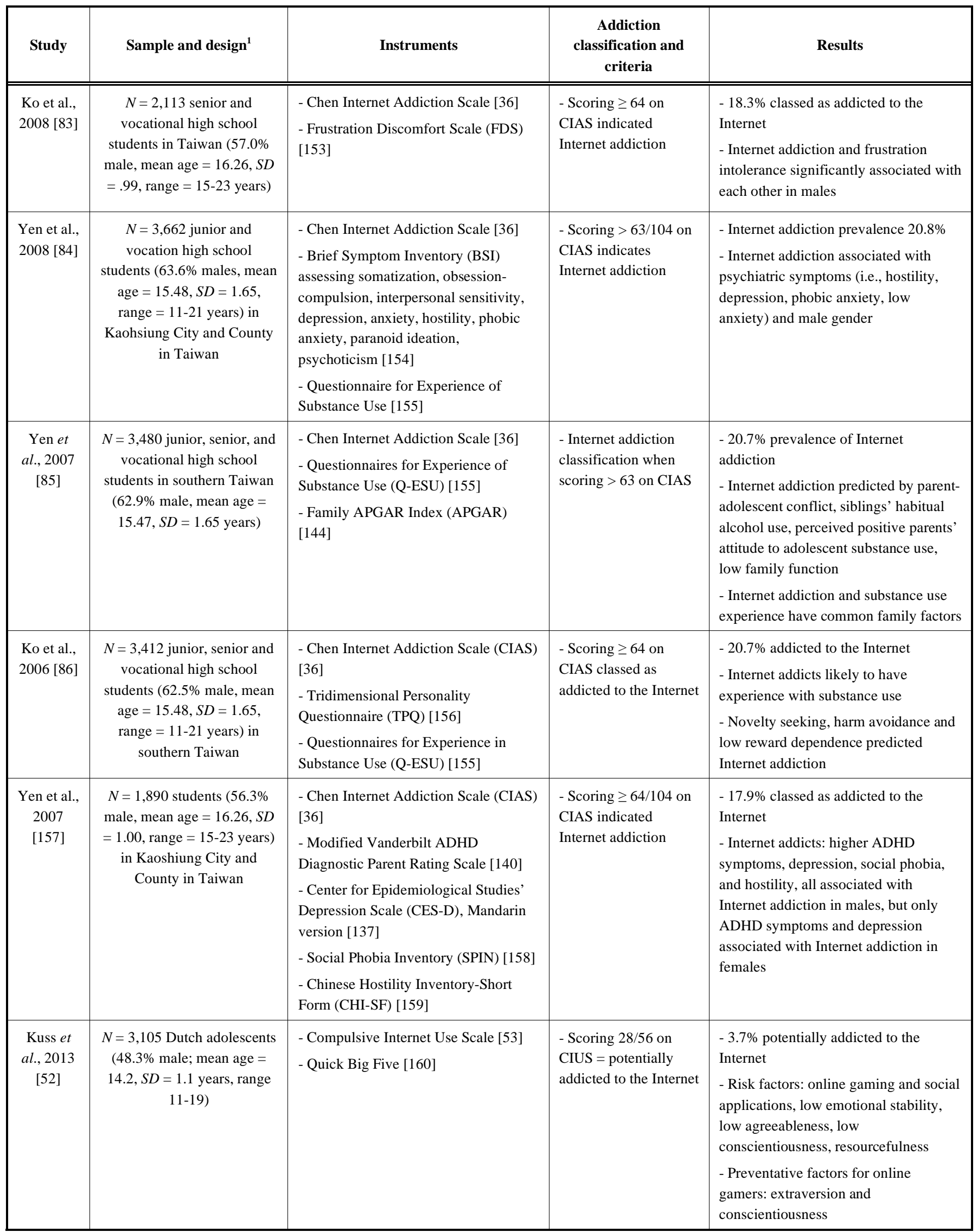


(Table 2) Contd....

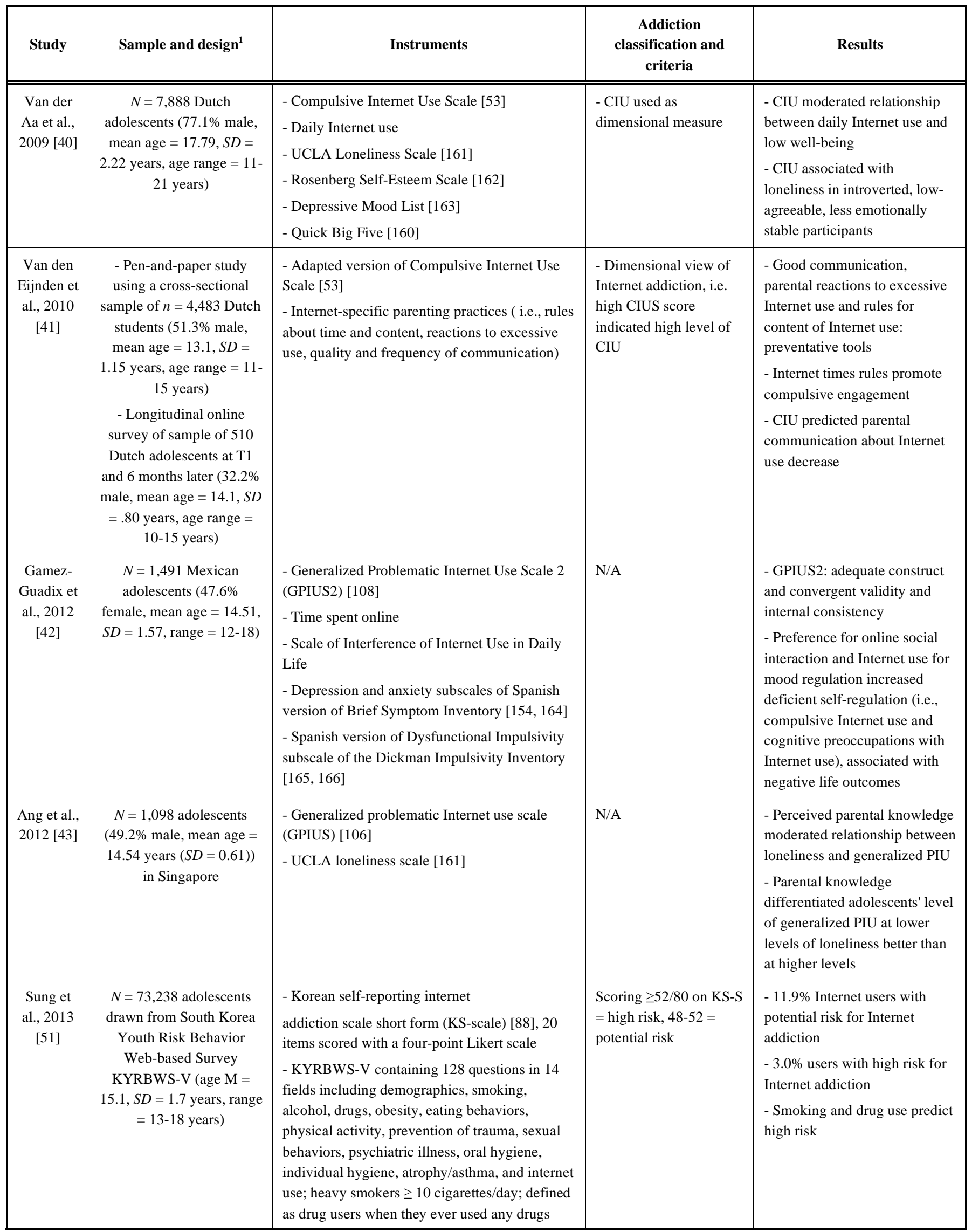


(Table 2) Contd....

\begin{tabular}{|c|c|c|c|c|}
\hline Study & Sample and design ${ }^{1}$ & Instruments & $\begin{array}{l}\text { Addiction classification } \\
\text { and criteria }\end{array}$ & Results \\
\hline $\begin{array}{l}\text { Lopez- } \\
\text { Fernandez } \\
\text { et al., } \\
2013 \text { [44] }\end{array}$ & $\begin{array}{c}N=1,131 \text { high school } \\
\text { students }(M=14.55, \\
S D=1.82, \text { range }=12 \text { and } 18 \\
\text { years }) \text { in Spain }\end{array}$ & $\begin{array}{l}\text { - Problematic Internet Entertainment } \\
\text { Use Scale for Adolescents (PIEUSA), } \\
\text { based on DSM-IV-TR criteria for } \\
\text { substance dependence and pathological } \\
\text { gambling disorders in adults; } \\
\text { diagnostic criteria proposed for IUD in } \\
\text { adults and adolescents and literature on } \\
\text { adolescent IUD prevalence. } \\
\text { - Scale contains } 30 \text { items rated on a 7- } \\
\text { point Likert scale, total score between } \\
\text { 30-210 }\end{array}$ & $\begin{array}{l}\text { PIEUSA highest score } \\
\text { representing the maximum } \\
\text { presence of the construct } \\
\text { under study over the last } 12 \\
\text { months }\end{array}$ & $\begin{array}{l}\text { - Prevalence of problematic } \\
\text { behaviour in Spanish adolescents } \\
\text { ca. } 5 \% \\
\text { - Scale was unidimensional, with } \\
\text { excellent internal consistency } \\
\text { (Cronbach's alpha of } 0.92 \text { ), good } \\
\text { construct validity, and positive } \\
\text { associations with alternative } \\
\text { measures of maladaptive Internet } \\
\text { use }\end{array}$ \\
\hline $\begin{array}{l}\text { Carbonell } \\
\text { et al., } \\
2012[46]\end{array}$ & $\begin{array}{c}N=1,879 \text { students in Spain } \\
(45.5 \% \text { males, mean age }= \\
15.5, S D=2.43 \text { years })\end{array}$ & $\begin{array}{l}\text { - Questionnaire on internet-related } \\
\text { experiences (CERI) [168] with two } \\
\text { factors, intra-and interpersonal } \\
\text { conflicts } \\
\text { - Questionnaire on cell phone related } \\
\text { experiences (CERM) [109] }\end{array}$ & $\begin{array}{l}\text { - Cluster analysis revealed } 3 \\
\text { groups; highest scoring } \\
\text { group ( } 26-40 \text { points) classed } \\
\text { as having frequent problems }\end{array}$ & $\begin{array}{l}\text { - } 6.1 \% \text { of sample had frequent } \\
\text { problems with Internet use with } \\
\text { no gender differences } \\
\text { - Specific Internet application } \\
\text { usages explained } 27.5 \% \text { of total } \\
\text { CERI variance }\end{array}$ \\
\hline $\begin{array}{l}\text { Sun et al., } \\
2012[47]\end{array}$ & $\begin{array}{c}\text { - Longitudinal study }(2 \\
\text { waves }) \\
-N=1,761 \text { students in China } \\
\text { (49\% male, mean age }=16.8, \\
\quad S D=.93 \text { years) } \\
-N=1,182 \text { students in the } \\
\text { USA }(57 \% \text { male, mean age }= \\
15.9, S D=.76 \text { years })\end{array}$ & $\begin{array}{l}\text { - Compulsive Internet Use Scale based } \\
\text { on Scale for Problematic Internet Use } \\
{[17,169]} \\
\text { - Frequency of cigarette smoking and } \\
\text { binge drinking in last } 30 \text { days }\end{array}$ & $\begin{array}{l}\text { - Scoring a mean of } 4 / 5 \text { on } \\
\text { CIUS as indicative of } \\
\text { Internet addiction }\end{array}$ & $\begin{array}{l}\text { - No relationship between CIU } \\
\text { and substance use at baseline } \\
\text { - CIU at baseline predicted } \\
\text { change in CIU and substance use } \\
\text { in females } \\
\text { - Substance use at baseline was } \\
\text { not predictive of CIU increase }\end{array}$ \\
\hline $\begin{array}{l}\text { Liu et al., } \\
2011[48]\end{array}$ & $\begin{array}{c}N=3,560 \text { high school } \\
\text { students in Connecticut, } \\
\text { USA (age range 14-18 years) }\end{array}$ & $\begin{array}{l}\text { - Demographics } \\
\text { - Risk behaviours } \\
\text { - Internet use } \\
\text { - Problematic Internet use }\end{array}$ & $\begin{array}{l}\text { - PIU modelled after } \\
\text { Minnesota Impulsive } \\
\text { Disorder Inventory and } \\
\text { defined as affirming } \\
\text { symptoms of craving, } \\
\text { withdrawal, and abstinence } \\
\text { attempts simultaneously }\end{array}$ & $\begin{array}{l}\text { - Prevalence of problematic } \\
\text { Internet use } 4 \% \\
\text { - PIU more common among } \\
\text { Asian and Hispanic students, } \\
\text { girls, associated with substance } \\
\text { use, depression, and aggression }\end{array}$ \\
\hline $\begin{array}{l}\text { Bener et } \\
\text { al., } 2011 \\
{[49]}\end{array}$ & $\begin{array}{c}\text { - Interviews based on } \\
\text { questionnaire } \\
-N=3000 \text { school students } \\
\text { (age range }=6-18 \text { years) in } \\
\text { Qatar }\end{array}$ & $\begin{array}{l}\text { - Excessive Internet use and television } \\
\text { viewing } \\
\text { - Vision and vision disorders } \\
\text { - Obesity and overnutrition }\end{array}$ & $\begin{array}{l}\text { - Excessive Internet use } \\
\text { defined as spending } \geq \\
\text { 3hours/day online }\end{array}$ & $\begin{array}{l}\text { - Obesity linked to online hours } \\
-1.9 \% \text { spent } \geq 3 \text { hours/day online, } \\
\text { were overweight/obese and had } \\
\text { low vision }\end{array}$ \\
\hline $\begin{array}{l}\text { Mythily } \\
\text { et al., } \\
2008[50]\end{array}$ & $\begin{array}{c}N=2,735 \text { adolescents in } \\
\text { Singapore }(49.3 \% \text { male } \\
\text { mean age }=13.9, S D=1.0 \\
\text { years })\end{array}$ & $\begin{array}{l}\text { - Sociodemographic questions } \\
\text { - Academic performance } \\
\text { - Social support and general wellbeing }\end{array}$ & $\begin{array}{l}\text { - Excessive Internet use = } \\
\text { using the Internet }>5 \\
\text { hours/day }\end{array}$ & $\begin{array}{l}\text { - } 17.1 \% \text { excessive Internet users } \\
\text { - Excessive Internet use related to } \\
\text { no rules regarding Internet use, } \\
\text { less confidants, sadness and } \\
\text { depression, poor academic } \\
\text { performance }\end{array}$ \\
\hline
\end{tabular}

Note 1. If not otherwise indicated, the study design was a cross-sectional survey. 
Table 3. Epidemiological Internet Addiction Studies in Adults.

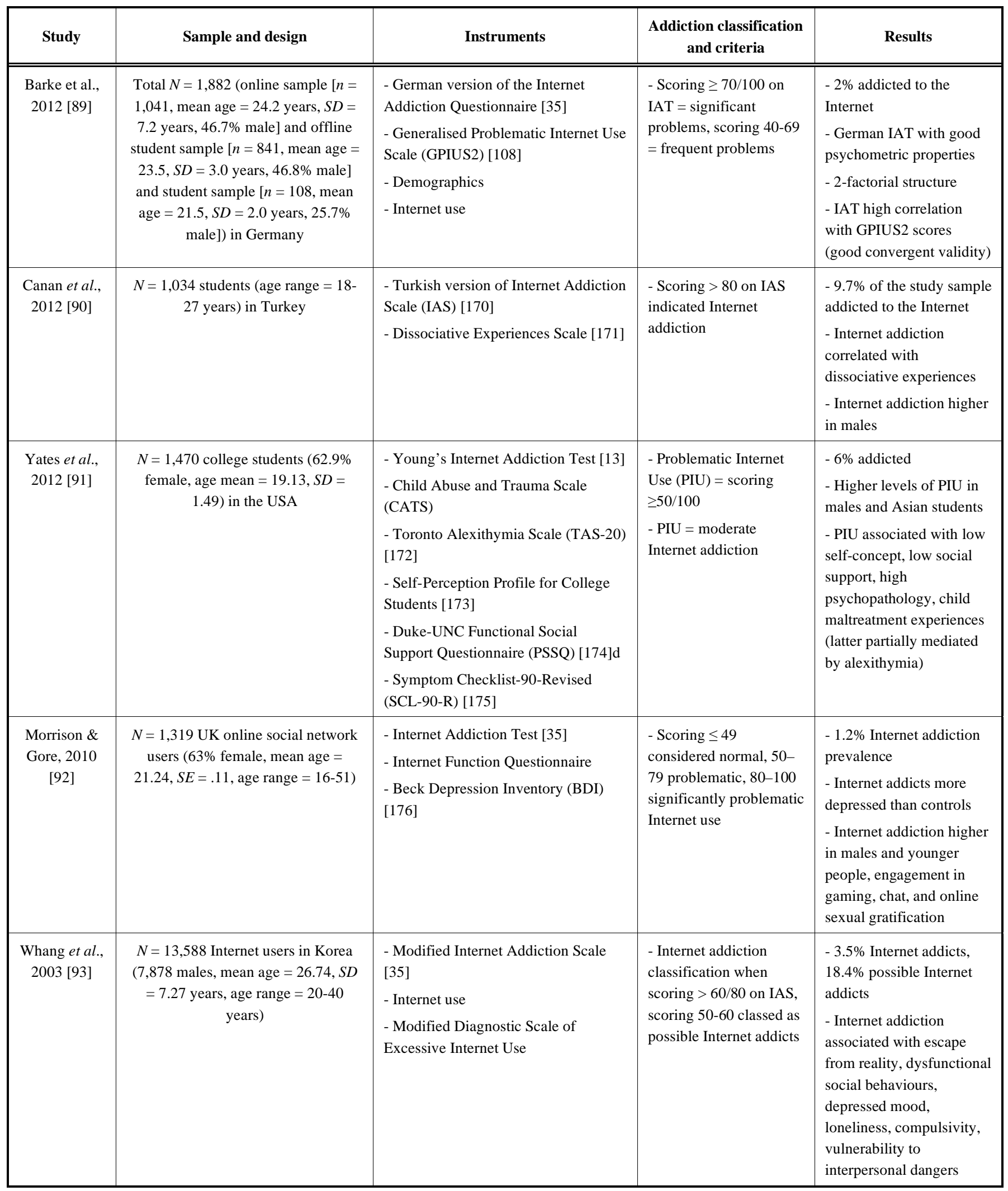


(Table 3) Contd....

\begin{tabular}{|c|c|c|c|c|}
\hline Study & Sample and design & Instruments & $\begin{array}{l}\text { Addiction } \\
\text { classification and } \\
\text { criteria }\end{array}$ & Results \\
\hline $\begin{array}{l}\text { Kheirkhah et } \\
\text { al., } 2010 \text { [95] }\end{array}$ & $\begin{array}{c}N=1,856 \text { Internet users } \\
\text { from Mazandaran } \\
\text { Province cities, Iran } \\
(50.9 \% \text { males, mean } \\
\text { age }=20.25, S D=4.19 \\
\text { years })\end{array}$ & $\begin{array}{l}\text { - Farsi version of Young's Internet Addiction } \\
\text { Questionnaire } \\
\text { - Time spent online }\end{array}$ & $\begin{array}{l}\text { - Endorsing } \geq 5 / 8 \\
\text { dichotomous items } \\
\text { was indicative of } \\
\text { Internet addiction }\end{array}$ & $\begin{array}{l}\text { - Internet addiction incidence } \\
=22.8 \% \\
\text { - Internet addicts spent more } \\
\text { time online than non-addicts } \\
\text { and used two-way } \\
\text { communication functions } \\
\text { (i.e., chat rooms) } \\
\text { - More male Internet addicts }\end{array}$ \\
\hline $\begin{array}{c}\text { Bakken } \text { et al., } \\
2009[96]\end{array}$ & $\begin{array}{l}N=3,399 \text { Norwegian } \\
\text { adults }(1,598 \text { male, age } \\
\text { range } 16-74 \text { years })\end{array}$ & $\begin{array}{l}\text { - Young Diagnostic Questionnaire (YDQ) [13] } \\
\text { - Subjective mental illness assessed with } 1 \text { question } \\
\text { each over last } 12 \text { months (i.e., sleep disorders, } \\
\text { depression, suicidal ideation, anxiety, obsession and } \\
\text { compulsions, alcohol/substance abuse) }\end{array}$ & $\begin{array}{l}\text { - Scoring } 5 / 8 \text { on } \\
\text { YDQ }=\text { Internet } \\
\text { addicts, scoring } 3-4= \\
\text { at risk users }\end{array}$ & $\begin{array}{l}\text { - Prevalence of Internet } \\
\text { addiction } 1.0 \%, 5.2 \% \text { at risk } \\
\text { users } \\
\text { - Highest prevalence in } \\
\text { young males } \\
\text { - Male gender, young age, } \\
\text { university level education, } \\
\text { and unsatisfactory financial } \\
\text { situation increased odds of } \\
\text { problematic Internet use } \\
\text { - YDQ score associated with } \\
\text { online time, sleeping } \\
\text { disorders, depression, and } \\
\text { other psychological problems }\end{array}$ \\
\hline $\begin{array}{l}\text { Lin et al., } \\
2011 \text { [98] }\end{array}$ & $\begin{array}{c}\text { Nationally } \\
\text { representative sample } \\
\text { of college students in } \\
\text { Taiwan }(N=3,616, \text { no } \\
\text { information about age } \\
\text { provided })\end{array}$ & $\begin{array}{l}\text { - Chen Internet Addiction Scale-Revised (CIAS-R) } \\
{[19,179]} \\
\text { - Positive Outcome Expectancy of Internet Use } \\
\text { Questionnaire [103] } \\
\text { - Refusal Self-Efficacy of Internet Use } \\
\text { Questionnaire [103] } \\
\text { - Ko's Depression Inventory (KDI) [180] } \\
\text { - Barratt Impulsiveness Scale Short-Form [181] - } \\
\text { Chinese Version of the Relationship Questionnaire } \\
\text { [182] } \\
\text { - Social Support Scale [104] }\end{array}$ & $\begin{array}{l}\text { - Internet addiction } \\
\text { measured via } 26 \\
\text { items: scoring }>67 \text { on } \\
\text { CIAS-R }\end{array}$ & $\begin{array}{l}\text { - Internet addiction } \\
\text { prevalence } 15.3 \% \\
\text { - Internet addiction correlated } \\
\text { with depressive symptoms, } \\
\text { Internet use positive outcome } \\
\text { expectancy, time spent } \\
\text { online, low refusal self- } \\
\text { efficacy of Internet use, } \\
\text { impulsivity, low academic } \\
\text { performance satisfaction, } \\
\text { male gender, insecure } \\
\text { attachment style }\end{array}$ \\
\hline
\end{tabular}


(Table 3) Contd....

\begin{tabular}{|c|c|c|c|c|}
\hline Study & Sample and design & Instruments & $\begin{array}{l}\text { Addiction classification } \\
\text { and criteria }\end{array}$ & Results \\
\hline $\begin{array}{l}\text { Yen et al., } \\
2011[99]\end{array}$ & $\begin{array}{c}N=2,262 \text { college students }(47.5 \% \\
\text { males, mean age }=20.77, S D=1.83 \\
\text { years }) \text { in Taiwan }\end{array}$ & $\begin{array}{l}\text { - Chen Internet Addiction Scale [36] } \\
\text { - Center for Epidemiological Studies' } \\
\text { Depression Scale [137, 146] } \\
\text { - Questionnaire for online activity } \\
\text { - Buss-Durkee Hostility Inventory, } \\
\text { Chinese version short form [142] }\end{array}$ & $\begin{array}{l}\text { Scoring } \geq 67 / 104 \\
\text { classified as Internet } \\
\text { addicts }\end{array}$ & $\begin{array}{l}\text { - No prevalence reported } \\
\text { - Hostility in real world } \\
\text { and online higher in } \\
\text { Internet addicts than } \\
\text { depressed individuals } \\
\text { - Internet addiction } \\
\text { associated with expressive } \\
\text { hostility behaviours }\end{array}$ \\
\hline $\begin{array}{l}\text { Tsai et al., } \\
2009[100]\end{array}$ & $\begin{array}{c}N=1,360 \text { university freshmen in } \\
\text { Taiwan }(69.6 \% \text { male })\end{array}$ & $\begin{array}{l}\text { - Chinese Internet Addiction Scale- } \\
\text { Revised (CIAS-R) [36] } \\
\text { - Chinese Health Questionnaire (CHQ- } \\
\text { 12) } \\
\text { [183] } \\
\text { - Measurement of Support Functions } \\
\text { (MSF) [184] } \\
\text { - Neuroticism subscale of the } \\
\text { Maudsley Personality Inventory (MPI) } \\
\text { [185] } \\
\text { - Lifestyle habits }\end{array}$ & $\begin{array}{l}\text { Scoring }>63 / 84 \\
\text { indicated Internet } \\
\text { addiction }\end{array}$ & $\begin{array}{l}\text { - } 17.9 \% \text { Internet addicts } \\
\text { - Male gender, } \\
\text { neuroticism, habit of } \\
\text { skipping breakfast, mental } \\
\text { health morbidity, deficien } \\
\text { social support, and CHQ } \\
\text { scores increased odds for } \\
\text { Internet addiction }\end{array}$ \\
\hline $\begin{array}{l}\text { Yen et al., } \\
2009[101]\end{array}$ & $\begin{array}{c}N=2,793 \text { students }(33.5 \% \text { male, } \\
\text { mean age }=20.46, S D=2.07 \text { years, } \\
\text { age range }=18-48 \text { years }) \text { in Taiwan }\end{array}$ & $\begin{array}{l}\text { - Chen Internet Addiction Scale [36] } \\
\text { - Adult ADHD Self-Report Scale } \\
\text { [186] } \\
\text { - Demographic questions }\end{array}$ & $\begin{array}{l}\text { Internet addiction when } \\
\text { scoring } \geq 68 / 104 \text { on } \\
\text { CIAS }\end{array}$ & $\begin{array}{l}\text { - } 12.9 \% \text { addicted to the } \\
\text { Internet } \\
\text { - Attention deficit and } \\
\text { impulsivity most strongly } \\
\text { related to Internet } \\
\text { addiction } \\
\text { - Attention deficit among } \\
\text { females more strongly } \\
\text { related to Internet } \\
\text { addiction }\end{array}$ \\
\hline $\begin{array}{l}\text { Lin } \text { et al., } \\
2008[103]\end{array}$ & $\begin{array}{c}N=4,456 \text { college students in Taiwan } \\
(46.7 \% \text { male, } \text { mean age }=19.87, S D \\
\quad=1.62 \text { years })\end{array}$ & $\begin{array}{l}\text { - Chen Internet Addiction Scale [36] } \\
\text { - Outcome Expectancy Questionnaire } \\
\text { - Refusal Self-Efficacy of Internet Use } \\
\text { Questionnaire (RSEIUQ) }\end{array}$ & $\begin{array}{l}\text { No information about } \\
\text { cut-off provided }\end{array}$ & $\begin{array}{l}\text { - No prevalence reported } \\
\text { - Refusal self-efficacy of } \\
\text { Internet use negatively, } \\
\text { and positive outcome } \\
\text { expectancy positively } \\
\text { predicted Internet } \\
\text { addiction, negative } \\
\text { outcome expectancy } \\
\text { predicted Internet } \\
\text { addiction via refusal self- } \\
\text { efficacy of Internet use }\end{array}$ \\
\hline
\end{tabular}


(Table 3) Contd....

\begin{tabular}{|c|c|c|c|c|}
\hline Study & Sample and design & Instruments & $\begin{array}{c}\text { Addiction classification } \\
\text { and criteria }\end{array}$ & Results \\
\hline $\begin{array}{l}\text { Yeh et al., } \\
2008[104]\end{array}$ & $\begin{array}{c}N=3,477 \text { college students in Taiwan } \\
(45 \% \text { male, mean age }=22.45, S D= \\
1.56 \text { years })\end{array}$ & $\begin{array}{l}\text { - Chen Internet Addiction Scale [36] } \\
\text { - Social Support Scale } \\
\text { - Virtual Social Support Scale } \\
\text { - Ko's Depression Inventory [188] }\end{array}$ & $\begin{array}{l}\text { No information about } \\
\text { scoring provided }\end{array}$ & $\begin{array}{l}\text { - No prevalence reported } \\
\text { - Actual and virtual social } \\
\text { support predicted Internet } \\
\text { addiction and were } \\
\text { mediated by depressive } \\
\text { symptoms in females } \\
\text { - Virtual social support } \\
\text { mediated and not } \\
\text { mediated by depressive } \\
\text { symptoms predicted } \\
\text { Internet addiction in males }\end{array}$ \\
\hline $\begin{array}{l}\text { Meerkerk et } \\
\text { al., } 2009 \text { [53] }\end{array}$ & $\begin{array}{c}\text { Representative samples of heavy } \\
\text { Dutch Internet users }(n \mathrm{~s}=447 \text { at } \mathrm{T} 1 \\
(49.4 \% \text { male, mean age }=38.5, S D= \\
12.5 \text { years }) \text { and } 229 \text { at } \mathrm{T} 2) \text { and a } \\
\text { convenience sample of regular } \\
\text { Internet users }(n=16,925,77.4 \% \\
\text { male, mean age }=25.3, S D=10.0 \\
\text { years) }\end{array}$ & $\begin{array}{l}\text { - Compulsive Internet Use Scale } \\
\text { (CIUS) } \\
\text { - Online Cognition Scale (OCS) [17] } \\
\text { - Time spent online } \\
\text { - Subjective problems }\end{array}$ & $\begin{array}{l}\text { Internet addiction } \\
\text { labelled "compulsive } \\
\text { Internet use" because } \\
\text { addicted to certain } \\
\text { activities not Internet } \\
\text { use per sé, leading to } \\
\text { compulsive use }\end{array}$ & $\begin{array}{l}\text { - No prevalence reported } \\
\text { - CIUS with good internal } \\
\text { consistency, good } \\
\text { concurrent and criterion } \\
\text { validity }\end{array}$ \\
\hline $\begin{array}{c}\text { Thatcher et } \\
\text { al., } 2008 \text { [54] }\end{array}$ & $\begin{array}{c}N=1,399 \text { technologically savvy } \\
\text { Internet users from South Africa } \\
(1,065 \text { males, aged between } 24-35 \\
\text { years })\end{array}$ & $\begin{array}{l}\text { - Problematic Internet Use } \\
\text { Questionnaire (PIUQ) [113] } \\
\text { - Distraction subscale of the online } \\
\text { cognition scale (OCS) [17] } \\
\text { - Modified version of Flow Scale } \\
\text { [190] }\end{array}$ & N/A & $\begin{array}{l}\text { - No prevalence reported } \\
\text { - Strong relationship } \\
\text { between problematic } \\
\text { Internet use, online } \\
\text { procrastination and online } \\
\text { flow }\end{array}$ \\
\hline $\begin{array}{c}\text { Demetrovics } \\
\text { et al., } 2008 \\
{[55]}\end{array}$ & $\begin{array}{c}N=1,037 \text { participants in Hungary } \\
(54.1 \% \text { male, } \text { mean age }=23.3, S D= \\
9.1 \text { years })\end{array}$ & $\begin{array}{l}\text { - Problematic Internet Use } \\
\text { Questionnaire (PIUQ) based on } \\
\text { Internet Addiction Questionnaire [115] } \\
\text { and Internet Addiction test [35] }\end{array}$ & $\begin{array}{l}\text { - Significantly } \\
\text { problematic Internet use } \\
=\text { scoring }>2 S D \text { s above } \\
\text { the mean on PIUQ } \\
\text { - Problematic Internet } \\
\text { use = scoring between } 1 \\
\text { and } 2 S D \text { above mean on } \\
\text { PIUQ }\end{array}$ & $\begin{array}{l}\text { - } 4.3 \% \text { with significant } \\
\text { problems because of } \\
\text { Internet use, } 10.1 \% \text { with } \\
\text { problems } \\
\text { - PIUQ with good internal } \\
\text { consistency, test-retest } \\
\text { reliability } \\
\text { - Most problematic } \\
\text { Internet users with high } \\
\text { male proportion, live with } \\
\text { restructured family, are } \\
\text { single }\end{array}$ \\
\hline
\end{tabular}


(Table 3) Contd....

\begin{tabular}{|c|c|c|c|c|}
\hline Study & Sample and design & Instruments & $\begin{array}{c}\text { Addiction classification } \\
\text { and criteria }\end{array}$ & Results \\
\hline $\begin{array}{l}\text { Cuhadar, } \\
2012[56]\end{array}$ & $\begin{array}{c}N=1,235 \text { students at a teacher } \\
\text { training programme in Turkey } \\
(30.0 \% \text { male })\end{array}$ & $\begin{array}{l}\text { - Problematic Internet Use Scale } \\
\text { [191] } \\
\text { - Social Interaction Anxiety Scale } \\
\text { [192] }\end{array}$ & $\begin{array}{l}\text { - PIUS ranges between } 33- \\
\text { 165, higher score indicates } \\
\text { less healthy Internet use, } \\
\text { stronger negative effects, } \\
\text { higher tendency for Internet } \\
\text { addiction pathology }\end{array}$ & $\begin{array}{l}\text { - No prevalence reported } \\
\text { - Internet use more } \\
\text { problematic for males } \\
\text { - Problematic Internet use } \\
\text { correlated with time spent } \\
\text { online } \\
\text { - Social interaction } \\
\text { anxiety predicted } \\
\text { problematic Internet use }\end{array}$ \\
\hline $\begin{array}{c}\text { Huang et al., } \\
2007 \text { [57] }\end{array}$ & $\begin{array}{c}N=1,029 \text { Chinese undergraduate } \\
\text { students in Beijing split into two: } n_{1}= \\
516(47.5 \% \text { male, mean age }=20.5, \\
S D=1.47, \text { range }=17-24 \text { years }), n_{2}= \\
513(46.2 \% \text { males, mean age }=20.7, \\
S D=1.51, \text { range }=17-24 \text { years }) \\
-N_{3}=67(27 \text { diagnosed Internet } \\
\text { addicts; } 31.3 \% \text { male, mean age }= \\
20.6, S D=.93 \text { years, range }=19-25 \\
\text { years })\end{array}$ & $\begin{array}{l}\text { - Chinese Internet Addiction } \\
\text { Inventory (CIAI) based on } \\
\text { Young's Internet Addiction Test } \\
{[35]}\end{array}$ & $\begin{array}{l}\text { Classification of Internet } \\
\text { addiction based on } 5+3 \\
\text { criteria [117] (i.e., endorsing } \\
\text { preoccupation, tolerance, } \\
\text { impulse control, mood } \\
\text { modification, and increasing } \\
\text { usage, and min. one of } \\
\text { conflicts, lying to others, } \\
\text { escaping from problems) }\end{array}$ & $\begin{array}{l}\text { - Subscale items with high } \\
\text { internal consistency and } \\
\text { acceptable test-retest } \\
\text { reliability, criterion } \\
\text { validity }\end{array}$ \\
\hline $\begin{array}{l}\text { Bergmark et } \\
\text { al., } 2011 \text { [58] }\end{array}$ & $\begin{array}{c}N=1,147 \text { participants in Sweden } \\
(50.4 \% \text { male, } \text { mean age }=45, \text { range }= \\
15-94 \text { years })\end{array}$ & $\begin{array}{l}\text { - Demographics } \\
\text { - Internet related problems }\end{array}$ & Internet related problems & $\begin{array}{l}\text { - } 5 \% \text { spend } \\
>30 \text { hours/week online at } \\
\text { home } \\
\text { - } 40 \% \text { experience } \geq 1 \\
\text { problem because of } \\
\text { Internet use } \\
-1.8 \% \text { experience all } \\
\text { problems }\end{array}$ \\
\hline
\end{tabular}

\subsubsection{Factors Associated with Internet Addiction in Adolescents}

\subsubsection{Sociodemographic Variables}

In addition to the prevalence estimates reported, the studies have found that in adolescents, Internet addiction is associated with the following sociodemographic variables: higher family income levels [61, 63], male gender [45, 61-63, 65, 66, 81, 83], female gender [48], being left behind (i.e., abandoned), and migrant [68], living in rural areas [81], being in senior high school, and having a monthly spending over 100 RMB [45]. The discrepancy between findings concerning associations between Internet addiction and gender can possibly be explained by the measures utilised, i.e., Liu et al. [48] found that a higher percentage of girls reports excessive usage behaviours, which might be indicative of their increased problem awareness relative to boys. Also, uninvestigated cultural differences could be the reason for these controversial sociodemographic findings.

\subsubsection{Internet use Variables}

Moreover, the following Internet use variables were found to be associated with Internet addiction in adolescents: age of first exposure to the Internet [195], Internet use for entertainment purposes [63], frequency and length of Internet use [45, 63, 64, 76], Internet access at home [61], Internet usage at an Internet café [70, $78]$, the use of online gaming $[46,52,69,196]$, social applications [45, 46, 52], and other Internet applications [69]. In addition to this, parental guidance with regards to Internet behaviours was reported to be associated with Internet addiction, specifically little parental communication about Internet use, rules about Internet times [41], and a lack of rules regarding Internet use [50]. 


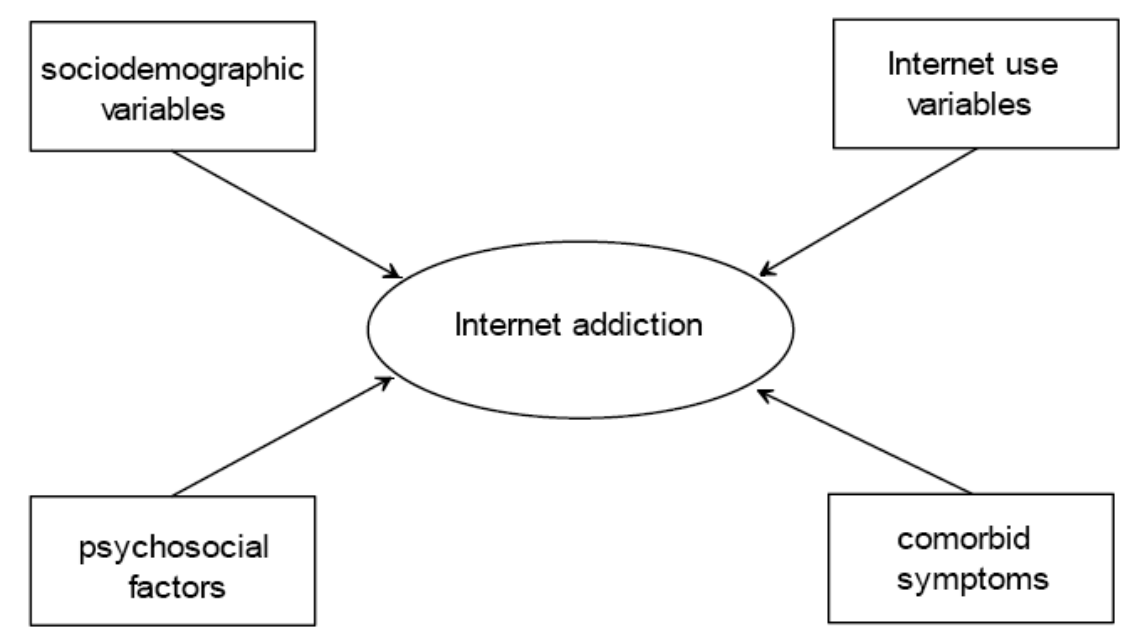

Fig. (1). Factors Associated with Internet Addiction.

\subsubsection{Psychosocial Factors}

Psychosocial factors have included internal characteristics such as Internet use for mood regulation [42], low life satisfaction [63, 78], low well-being [40], loneliness [43, 63], lack of confidants [50], preference for online social interaction, negative life outcomes [42]. Moreover, the following personality characteristics were reported: novelty seeking, harm avoidance, and low reward dependence [86], low self-esteem [78], frustration intolerance (particularly for males) [83], introversion [40], low agreeableness, low emotional stability [40, 52], low conscientiousness and resourcefulness [52]. In addition, social variables were social adaptation [63], stress [65], low academic achievement [45, 50], poor relations with school [65, 81], leisure boredom [75], breadth of extracurricular activities [78], peers and siblings who drink alcohol $[81,87]$. Furthermore, a variety of family variables were associated with Internet addiction. These included family conflict and dissatisfaction [64, 65, 81], parental bonding [69], perceived parental monitoring $[75,81]$, and perceived positive attitude to adolescent substance use by parents [157].

\subsubsection{Comorbid Symptoms}

Comorbid symptoms have included alcohol and substance use $[47,48,51,65,66,71,74,82,86]$, proneness for problem behaviours [82], depression [48, 50, 67, 68, 78, 81, 83, 87, 124], suicidal ideation [124][105], ADHD [67, 79, 157], social phobia and phobic anxiety $[79,84,87]$, schizophrenia, obsessive-compulsive disorder [67], psychoticism [71], and antisocial/aggressive behaviours [48, $72,79,80,87]$. Finally, a number of psychosomatic problems have been linked to problematic Internet use, such as a poor health condition, excessive daytime sleepiness [66], lack of energy, physiological dysfunction, weakened immunity [63], obesity, and poor vision [49].

\subsubsection{Factors Associated with Internet Addiction in Adults 3.3.2.1. Sociodemographic Variables}

A number of factors have been found to be associated with Internet addiction in adults. With regards to sociodemographic variables, this includes male gender [55, 56, 90, 92, 95-98, 100], younger age [92, 96], city residence [94], single parent and restructured family [55, 94], being single [55], financial difficulties, university level education [96], as well as Asian ethnicity [91] were reported.

\subsubsection{Internet use Variables}

Various Internet use variables have been found to be associated with Internet addiction. These include increased time spent online
[56, 58, 59, 95, 96, 98], early Internet exposure [94], positive outcome expectancy, low refusal self-efficacy of Internet use [98, 103], online flow and online procrastination [54]. Furthermore, the usage of a number of different Internet applications has been linked to Internet addiction. These include social applications [60, 92, 95], online gaming and other applications [59,92], and a combination of online gaming and openness to experience [60].

\subsubsection{Psychosocial Factors}

A number of psychosocial factors were found to be associated with Internet addiction. Psychological variables have included impulsivity [98, 101], neuroticism [60, 100], low agreeableness [60], low self-concept [91], escapism, loneliness [93], fun-seeking [102], and negative emotion avoidance [59]. Social variables were low satisfaction with academic performance [97, 98], an insecure attachment style [98], child maltreatment experiences [91], low social support [91, 100], lack of family love [97], homesickness [94], virtual social support directly and indirectly via depressive symptoms, and low actual social support directly and indirectly via depressive symptoms [104]. In addition to this, dysfunctional social behaviours and vulnerability to interpersonal dangers [93] were reported. Moreover, a behavioural factor, i.e., a habit of skipping breakfast [100] was statistically associated with Internet addiction.

\subsubsection{Comorbid Symptoms}

In terms of comorbid symptoms, the following factors have been found: depression [92-94, 97, 98, 104], anxiety [94, 96], harmful alcohol use [102], compulsivity [93], sleeping disorders [96], ADHD [101], hostility [99], dissociative experiences and depersonalisation [59, 90], psychological problems and high psychopathology in general $[91,96,100]$.

\section{DISCUSSION}

The aim of this paper was to review and describe epidemiological Internet addiction research since the millennium. The conceptualisation of Internet addiction was assessed by inquiring into commonly utilised assessment tools. Based on this, the prevalence rates in the studies to date have been identified, and the associated factors highlighted. Overall, this literature review supports conclusions about the Internet addiction research field that have been made previously $[22,23,25]$, indicating that Internet addiction assessment is inauspiciously varied. This literature review has highlighted that to date, no gold standard for Internet addiction diagnosis and assessment exists.

A sum total of 21 different Internet addiction questionnaires have been identified in this review, some of which use criteria as 
indistinctive as the number of problems experienced by individuals [59], or the number of hours spent on the Internet as being suggestive of Internet addiction problems [49, 50]. Moreover, other studies $[42,43,53]$ used dimensional measures that do not allow for the assessment of prevalence rates. If Internet addiction research aims to run parallel to actual clinical assessment, standardised cutoffs need to be implemented so that findings can be compared and disseminated [197]. The label "Internet addiction" inherently refers to a psychopathology, a diagnosable clinical entity, which is capable of distinguishing individuals that are not affected (i.e., not addicted) from those that are addicted to using the Internet, thus denoting diagnostic sensitivity and specificity [198]. If the nosology of addiction is used, its usage must be justified against actual presenting problems in clinical settings and thus clinical utility is called for in assessment scales.

In addition to the wide variability in diagnostic tools used for Internet addiction assessment, the most commonly used scales [13, $35,36]$ suffer from a variety of shortcomings. First of all, none of Young's [13, 35] measures include a time criterion. If Internet addiction was to be treated as a behavioural analogue to substance dependence [105], a minimum number of symptoms need to be present simultaneously over the same 12-month period. Using Young's criteria, an individual would be classed as Internet addict when he or she experienced five of eight symptoms altogether over their lifetime, evidently questioning the clinical validity of the diagnostic construct. Moreover, the binary response format in the IADQ [13] is very limited with regards to the amount of information utilised relative to a dimensional assessment of symptom presence [199]. The lack of a temporal dimension and binary scoring is likely to lead to inflated prevalence rates of Internet addiction [22]. A dimensional approach to symptom evaluation can offer a "more valid description of psychopathology" [200] as it overcomes the limitations of categorical approaches. The CIAS [36], on the other hand, includes items such as time management to assess the degree of Internet use-related problems. It is questionable to what extent variables like time management constitute a criterion that is relevant for diagnosis, and therefore, the Internet addiction prevalence rates reported using the CIAS may be an overestimate. In light of this, the time management criterion could be combined with the reason for Internet use being either instrumental (i.e., information seeking) or ritual (i.e., entertainment) [201], as it appears not to be the time that constitutes the criterion, but the time combined with the usage motivation which distinguishes Internet use from potential abuse.

In this literature review, it has been found that prevalence rates are particularly diverse across samples and across measurement instruments, indicating a relatively low validity across studies. The prevalence rates for adolescents ranged between $0.8 \%$ in Italy [62] and $13.8 \%$ in South Korea [67] assessed via the IAT, between $1.4 \%$ in Finnish girls [77] and 26.7\% of adolescents in Hong Kong [73] using the IADQ, and between $10.8 \%$ in Southern Taiwan [79] and $22.5 \%$ of boys under 15 in Taiwan [81] assessed via the CIAS. Using miscellaneous criteria and instruments, the Internet addiction prevalence varied between 3.0\% in South Korea [51] and $17.1 \%$ in Singapore [50]. Similar divergence was found in the adult samples. Prevalence rates varied between $1.2 \%$ of Internet users in the UK [92] and 9.7\% of College students in Turkey [90] assessed via the IAT, between $1.0 \%$ in Norwegian adults [96] and $22.8 \%$ of Iranian Internet users [95] as evaluated using the IADQ, and between $12.3 \%$ [102] and $17.9 \%$ of college first-years in Taiwan [100] using the CIAS. Finally, the usage of miscellaneous criteria in adults revealed that between $1.8 \%$ of Swedish adults [58] and $4.3 \%$ of Hungarian adults [55] experience significant problems because of their Internet use.

Taken together, the dissimilar prevalence rates reported can thus partially be attributed to different classification criteria used, more so than differences between age groups (i.e., adolescents and adults). Internet addiction (or Internet-use related symptoms) appears to be prevalent across the age spectrum, as both adolescent and adult groups seem to experience associated problems. Another reason for different prevalence rates concerns the population studied, as conceivably there may exist differences between general populations and Internet users specifically. Similarly, some studies used convenient online sampling [e.g., 60], which allows for the collection of a large pool of data [202], but might introduce a sampling bias. Moreover, the cultural context of the studies must be attended to as measurement instruments are not universal and mental problems are experienced and reported in different ways across various cultures [203].

In addition to this, although in some studies the same scales have been used, different cut-off criteria have been adopted. Presumably, the severity of Internet-addiction related symptoms in a person scoring 50 on a 100-point scale is lower than for a person scoring 80 on the same scale. This needs to be borne in mind when evaluating actual prevalence. The use of cut-off points is common practice in clinical evaluation of patients. The diagnostic manuals used in clinical practice today, i.e., the DSM IV-TR [105] and the ICD-10 [112] use cut-offs based on which the presence of symptoms can be evaluated from a clinical point of view. The usage of commonly agreed upon cut-off points for mental disorder diagnosis primarily serves the purpose of clinical utility by facilitating diagnosis, medical record keeping, and clinical research (such as meta-analyses) relative to a potential dimensional approach [197].

Moreover, the self-report survey methodology (rather than actual clinical assessment by specially qualified professionals) necessitates additional caution in the evaluation of results. However, in favour of self-reports, it needs to be stated that their usage is very common in psychological research as it has a number of advantages over other methods, such as interviews [204]. Surveys reduce interviewer bias, are anonymous, allow for considered answers rather than immediate responses, and are accessible to wider samples. Furthermore, they are more cost-effective as they do not require professional training for administration. The disadvantages include simple (and thereby possibly limited) questions, lack of probing, limited control, and a relatively low response rate [204]. In terms of the applicability of self-reports in clinical assessment situations, it has been found that there does not appear to be a significant difference between self-rating and clinical rating with regards to major depressive disorder [205], suggesting that self-reports may realistically reflect the symptom experience of individuals suffering from psychopathology. In addition to this, the usage of psychometric tools to evaluate patients' mental health status prior to treatment initiation is common practice in modern clinical psychology [206, 207]. Moreover, it has been shown that self-reported symptom severity distinguished medical outpatients with mental disorders significantly from patients without [208], supporting the usefulness of self-reports for initial psychiatric evaluation of a patient's mental health status. This indicates that self-reports are a valuable tool in initial psychopathology assessment.

Various additional factors have been specified as statistically related to Internet addiction, namely sociodemographic, Internet use, and psychosocial variables, as well as comorbid symptoms and disorders. The most common sociodemographic variable associated with Internet addiction was male gender both in adolescents [e.g., 65,81 ] as well as in adults [e.g., 96, 98]. Research suggests that the link between male gender and Internet addiction may be mediated by other variables, such as the type of online application used. For instance, males have preferences for online activities that are more frequently dysfunctional, such as online games and online sex [24]. Moreover, the higher prevalence reported in males could be mediated by individual differences in personality traits, such as low self-control, impulsivity and sensation seeking [209, 210]. Results of the National Comorbidity Survey indicated that adolescent males 
have been identified to be $30 \%$ to $80 \%$ more at risk for developing substance-related disorders than adolescent females [211], suggesting that they might be more vulnerable to addictions generally.

Internet use variables have been investigated with the most commonly identified link being between Internet addiction and time spent online [e.g., 45, 95, 98] and the use of specific online applications, notably gaming and social applications $[45,46,52,60$, 92]. The time spent online may be a tentative indicator of an increasing tolerance to using the Internet, which is a core criterion for substance dependence [105]. The studies presented however mainly used cross-sectional data, which do not provide for an evaluation of a potential progression of online times. This indicates that the statistical associations between time spent online and Internet addiction symptoms appear as short-cut for appraising the presence of tolerance. In addition to this, time that is spent online is time that is not spent offline, i.e., engaging in alternative recreational activities, and spending time with friends and significant others, which can lead to significant problems and potentially cause impairment. Moreover, factors related to the virtual context of Internet use may furthermore play a special role in the development of potentially addictive behaviours. In a recent study, it was found that flow (i.e., a state where the activity at hand matches challenge and skill [212] and telepresence (i.e., the perception of being present in the virtual environment [213] were predictive of Internet abuse [214].

Nevertheless, it still appears relatively unclear what individuals get addicted to as online, they can engage in a multitude of behaviours, possibly with different consequences. Previous reviews have indicated that the engagement in specific behaviours on the Internet may lead to symptoms associated with addiction, such as online gaming [215] and online social networking [216], suggesting that certain behaviours on the Internet might be more problematic than others. Online gaming has been identified as potentially addictive in previous research, highlighting that certain personality traits, self-regulation capacities, gaming motivations, as well as structural game characteristics may increase the risk for online gaming addiction [215]. The literature base for Internet social application addiction, relative to gaming, is diminutive, however, the usage of social networking sites has been found to be potentially addictive as overuse can result in a variety of negative consequences for the individual [216].

This review has moreover shown that certain psychosocial problems associated with Internet addiction may prove fruitful in distinguishing between adolescent and adult Internet related problems. For instance, in some studies using adolescents, various forms of family conflicts and problems have been noted [64, 65, 81], indicating that stressors in the realm of family may reinforce the excessive engagement with the Internet as a form of dysfunctional coping. A secure and supportive family environment thus appears to be particularly relevant for adolescents as the lack of it increases the risk for Internet addiction. In addition to this, poor academic achievement was noted in a variety of studies as risk factor for Internet addiction [e.g., 50, 97, 217]. Scholastic and academic pressures (particularly in Asian countries) appear to have a negative influence on the adolescents' and young adults' adjustment and life satisfaction which again may lead them to seek refuge in online worlds by applying a dysfunctional coping strategy. Furthermore, from the analysis, it appeared that in general, certain personality characteristics (e.g., impulsivity, neuroticism) may put individuals at risk for developing Internet addiction. That is, specific individual factors may increase the vulnerability for Internet addiction, which, possibly in the presence of external stressors such as poor family function and/or insufficient academic achievement, may potentiate the risk for Internet addiction relative to individuals with none or fewer such predisposing factors.

The presence of a variety of comorbid symptoms and disorders as well as psychosocial and psychosomatic problems indicates that
Internet addiction does not occur in a vacuum. Presumably, the Internet is used in order to cope with problems (e.g., loneliness, family conflict, depressive symptoms), which in turn may exacerbate potential Internet addiction symptoms [e.g., 218, 219]. Similar relationships between alcohol use for coping purposes and alcohol abuse have been established [220], suggesting that the link between everyday problems and Internet use as coping mechanism appear viable. It could be possible that in some cases, rather than being a psychopathology per se, the excessive use of the Internet could be used as (dysfunctional) coping mechanism to deal with primary disorders, such as depression or Post-Traumatic Stress Disorder. Future research is required to more closely pay attention to these potentialities and to establish the theoretical framework for Internet addiction, which as yet appears to be somewhat limited.

Moreover, research indicates that gaming addiction can appear as both, a primary and a secondary disorder [221], suggesting that the same may hold true for other forms of addictive online behaviours. In general, the comorbidity between mental disorders and addiction is high [222], with individuals suffering from mental health problems three times more likely to be addicted relative to healthy populations [223]. The widely reported co-existence of Internet addiction and substance use/abuse suggests that addictions share etiological mechanisms, such as neurobiological and psychosocial factors [33], supporting the syndrome model of addiction [224]. Overall, investigating associated factors allows for the identification of populations at risk for developing Internet addiction. These can be specifically targeted by prevention campaigns as well as specialised health care initiatives.

As regards the different classifications adopted in the studies reviewed, one could argue that the construct of Internet addiction has been created from an atheoretical perspective. Scholars have conceptualized the problematic use of the Internet in the framework of substance abuse a priori following the DSM-IV-TR criteria [105], and developed assessment tools based on these criteria. This approach to Internet addiction classification and assessment is problematic as it lacks a theoretical basis and may oversimplify the issue. In light of this, research is required which assesses the biopsychosocial processes that contribute to the development of addictive behaviours on the Internet. Discerning similarities in brain activity and structural abnormalities across addictions including Internet addiction seems to be a first step on the way towards understanding Internet addiction more fully [31]. Although the reported prevalence rates vary, they suggest that an Internet-use related disorder exists.

\section{LIMITATIONS AND SUGGESTIONS FOR FUTURE RESEARCH}

A number of limitations need to be addressed. First, the goal of a review of epidemiological studies of Internet addiction precluded the inclusion of (i) studies of smaller sample sizes and (ii) studies specifically focusing on online gaming addiction. These have been discussed in detail elsewhere $[215,225]$ and were therefore left out of the present analysis. Future research should contrast and compare the studies assessing Internet addiction and addictions to specific Internet activities. This will shed additional light upon the APA's decision to limit the new preliminary diagnostic category of addictive Internet use to games [20]. Second, the current literature review does not contain specific information about the respective number of studies excluded due to not meeting the required inclusion criteria. In future studies, it is advised to pay closer attention to the frequency of exclusion as per specified criterion.

\section{CONCLUSION}

In sum, the present literature review of epidemiological empirical Internet addiction research has shown that there are problems in the assessment of Internet addiction. No clear gold standard exists based on which the status and severity of Internet 
addiction symptoms can be evaluated, and neither is the usage of current tools standardised in such a way that cross-study comparisons are facilitated. In light of this, it is recommended that (i) a clearly defined nosology of Internet addiction is established as clear-cut psychopathological entity, (ii) a single denomination for this entity is adopted, and (iii) to standardise the assessment tools and procedures to ensure high clinical utility. Internet addiction has been named an "important global mental health problem" [29], as Internet use-related problems and associated addiction symptoms have been reported on a global scale throughout adolescence and adulthood. Clearly, there is a need for nosological precision so that ultimately those in need can be helped by translating the scientific evidence established in the context of Internet addiction into actual clinical practice.

\section{CONFLICT OF INTEREST}

The authors confirm that this article content has no conflicts of interest.

\section{ACKNOWLEDGEMENTS}

Declared none.

\section{REFERENCES}

[1] International Telecommunication Union. Internet users.: International Telecommunication Union; 2012 [21.11.2012]; Available from: http: //www.itu.int/ITU-D/ict/statistics/index.html.

[2] International Telecommunication Union. The world in 2013. ICT facts and figures. Geneva, Switzerland: International Telecommunication Union2013.

[3] The Nielsen Company. State of the media: U.S. digital consumer report. The Nielsen Company 2012.

[4] The Nielsen Company. Australian online landscape review. The Nielsen Company2012.

[5] Soper WB, Miller MJ. Junk-time junkies: An emerging addiction among students. School Counselor1983; 31(1): 40-3.

[6] Goldberg I. Internet Addictive Disorder (IAD) diagnostic criteria. 1996 [17.04.2013]; Available from: http: //www.webcitation.org/query?url=http $\% 3 \mathrm{~A} \% 2 \mathrm{~F} \% 2 \mathrm{Fwww} . p s y c o m$. net $\% 2$ Fiadcriteria.html\&date $=2013-02-06$.

[7] American Psychiatric Association. Diagnostic and Statistical Manual for Mental Disorders IV. Washington, D. C.: American Psychiatric Association; 1994

[8] Young KS. Addictive use of the Internet: A case that breaks the stereotype. Psychological Reports 1996; 79: 899-902.

[9] Griffiths MD. Internet "addiction": An issue for clinical psychology? Clinical Psychology Forum1996; 97: 32-6.

[10] Young KS. Psychology of computer use: XL. Addictive use of the Internet: A case that breaks the stereotype. Psychological Reports1996 Dec; 79(3): 899-902.

[11] Rheingold $H$. The virtual community: Homesteading on the electronic frontier. Cambridge, Massachusetts: MIT; 1993.

[12] Griffiths MD. Internet addiction: An issue for clinical psychology? Clinical Psychology Forum1996; 97: 32-6.

[13] Young K. Internet addiction: The emergence of a new clinical disorder. Cyberpsychology \& Behavior1998; 3: 237-44.

[14] Black DW, Belsare G, Schlosser S. Clinical features, psychiatric comorbidity, and health-related quality of life in persons reporting compulsive computer use behavior. Journal of Clinical Psychiatry1999 Dec; 60(12): 839-44.

[15] te Wildt BT. Internet dependency: Symptoms, diagnosis and therapy. In: Cornelius K, Hermann D, editors. Virtual worlds and criminality. Berlin: Springer; 2011. p. 61-78.

[16] Morahan-Martin J, Schumacher P. Incidence and correlates of pathological Internet use among college students. Computers in Human Behavior2000; 16(1): 13-29.

[17] Davis RA, Flett GL, Besser A. Validation of a new scale for measuring problematic Internet use: Implications for preemployment screening. Cyberpsychology \& Behavior2002 Aug; 5(4): 331-45

[18] Greenfield DN. Virtual addiction. Oakland, CA: New Harbinger; 1999.
[19] Ko CH, Yen JY, Chen CC, Chen SH, Yen CF. Proposed diagnostic criteria of Internet addiction for adolescents. Journal of Nervous and Mental Disease2005 Nov; 193(11): 728-33.

[20] American Psychiatric Association. Diagnostic and Statistical Manual of Mental Disorders (DSM-5). Arlington, VA: American Psychiatric Association; 2013.

[21] Petry NM, O'Brien CP. Internet Gaming Disorder and the DSM-5. Addiction2013; 108(7): 1186-7.

[22] Widyanto L, Griffiths M. "Internet addiction": A critical review. International Journal of Mental Health and Addiction2006; 4: 31 51 .

[23] Byun S, Ruffini C, Mills JE, Douglas AC, Niang M, Stepchenkova S, Lee SK, Loutfi J, Lee JK, Atallah M, Blanton M. Internet addiction: Metasynthesis of 1996-2006 quantitative research CyberPsychology \& Behavior2009 Apr; 12(2): 203-7.

[24] Chou C, Condron L, Belland JC. A review of the research on Internet addiction. Educational Psychology Review2005 Dec; 17(4): 363-88.

[25] Beard KW. Internet addiction: A review of current assessment techniques and potential assessment questions. Cyberpsychology \& Behavior2005 Feb; 8(1): 7-14

[26] Liu C, Liao M, Smith DC. An empirical review of Internet addiction outcome studies in China. Research on Social Work Practice2012 May 1, 2012; 22(3): 282-92.

[27] King DL, Delfabbro PH, Griffiths MD, Gradisar M. Assessing clinical trials of Internet addiction treatment: A systematic review and CONSORT evaluation. Clinical Psychology Review2011 Nov; 31(7): 1110-6.

[28] Winkler A, Dorsing B, Rief W, Shen Y, Glombiewski JA Treatment of internet addiction: A meta-analysis. Clinical Psychology Review2013 Mar; 33(2): 317-29.

[29] Ko CH, Yen JY, Yen CF, Chen CS, Chen CC. The association between Internet addiction and psychiatric disorder: A review of the literature. European Psychiatry2012 Jan; 27(1): 1-8.

[30] Billieux J, Van der Linden M. Problematic use of the Internet and self-regulation: A review of the initial studies. The Open Addiction Journal2012; 5(Suppl 1: M4): 24-9.

[31] Kuss DJ, Griffiths MD. Internet and gaming addiction: A systematic literature review of neuroimaging studies. Brain Sciences2012; $2: 347-74$

[32] Lortie CL, Guitton MJ. Internet addiction assessment tools: Dimensional structure and methodological status. Addiction2013; 108(7): 1207-16.

[33] Griffiths MD. A "components" model of addiction within a biopsychosocial framework. Journal of Substance Use2005; 10: 191-7.

[34] Chiang IP, Su Y-H. Measuring and analyzing the causes of problematic Internet use. Cyberpsychology, Behavior and Social Networking2012 Nov; 15(11): 591-6.

[35] Young K. Caught in the net. New York: Wiley; 1998.

[36] Chen SH, Weng LC, Su YJ, Wu HM, Yang PF. Development of Chinese Internet Addiction Scale and its psychometric study. Chinese Journal of Psychology2003; 45: 279-94.

[37] Conti MA, Jardim AP, Hearst N, Cordás TA, Tavares H, Nabuco de Abreu C. Evaluation of semantic equivalence and internal consistency of a Portuguese version of the Internet Addiction Test (IAT). Revista De Psiquiatria Clínica2012; 39(3): 106-10.

[38] Stavropoulos V, Alexandraki K, Motti-Stefanidi F. Recognizing internet addiction: Prevalence and relationship to academic achievement in adolescents enrolled in urban and rural Greek high schools. Journal of Adolescence2013; 36(3): 565-76.

[39] Ko C-H, Yen J-Y, Yen C-F, Chen C-C, Yen C-N, Chen S-H. Screening for internet addiction: An empirical study on cut-of points for the Chen internet addiction scale. Kaohsiung Journal of Medical Sciences2005 Dec; 21(12): 545-51.

[40] van der Aa N, Overbeek G, Engels RCME, Scholte RHJ, Meerkerk GJ, van den Eijnden RJJM. Daily and compulsive Internet use and well-being in adolescence: A diathesis-stress model based on Big Five personality traits. Journal of Youth and Adolescence2009; 38 765-76.

[41] van den Eijnden RJ, Spijkerman R, Vermulst AA, van Rooij AJ, Engels RCME. Compulsive Internet use among adolescents: Bidirectional parent-child relationships. Journal of Abnormal Child Psychology2010; 38: 77-89.

[42] Gamez-Guadix M, Villa-George FI, Calvete E. Measurement and analysis of the cognitive-behavioral model of generalized 
problematic Internet use among Mexican adolescents. Journal of Adolescence2012 Dec; 35(6): 1581-91.

[43] Ang RP, Chong WH, Chye S, Huan VS. Loneliness and generalized problematic Internet use: Parents' perceived knowledge of adolescents' online activities as a moderator. Computers in Human Behavior2012 Jul; 28(4): 1342-7.

[44] Lopez-Fernandez O, Freixa-Blanxart M, Luisa Honrubia-Serrano M. The Problematic Internet Entertainment Use Scale for Adolescents: Prevalence of problem Internet use in Spanish high school students. Cyberpsychology, Behavior and Social Networking2013 Feb; 16(2): 108-18.

[45] Xu J, Shen L-x, Yan C-h, Hu H, Yang F, Wang L, Kotha SR, Zhang L-n, Liao X-p, Zhang J, Ouyang F-x, Zhang J-s, Shen X-m. Personal characteristics related to the risk of adolescent internet addiction: A survey in Shanghai, China. BMC Public Health2012 Dec 22; 12: 1106

[46] Carbonell X, Chamarro A, Griffiths M, Oberst U, Cladellas R, Talarn A. Problematic Internet and cell phone use in Spanish teenagers and young students. Anales De Psicologia2012 Oct; 28(3): 789-96.

[47] Sun P, Johnson CA, Palmer P, Arpawong TE, Unger JB, Xie B, Rohrbach LA, Spruijt-Metz D, Sussman S. Concurrent and predictive relationships between compulsive Internet use and substance use: Findings from vocational high school students in China and the USA. International Journal of Environmental Research and Public Health2012 Mar; 9(3): 660-73.

[48] Liu TC, Desai RA, Krishnan-Sarin S, Cavallo DA, Potenza MN. Problematic Internet use and health in adolescents: Data from a high school survey in Connecticut. Journal of Clinical Psychiatry2011 Jun; 72(6): 836-45.

[49] Bener A, Al-Mahdi HS, Ali AI, Al-Nufal M, Vachhani PJ, Tewfik I. Obesity and low vision as a result of excessive Internet use and television viewing. International Journal of Food Sciences and Nutrition2011 Feb; 62(1): 60-2.

[50] Mythily S, Qiu S, Winslow M. Prevalence and correlates of excessive internet use among youth in Singapore. Annals Academy of Medicine Singapore2008 Jan; 37(1): 9-14.

[51] Sung J, Lee J, Noh HM, Park YS, Ahn EJ. Associations between the risk of Internet addiction and problem behaviors among Korean adolescents. Korean Journal of Family Medicine2013; 34(2): 11522

[52] Kuss DJ, van Rooij A, Shorter GW, Griffiths MD, van de Mheen D. Internet addiction in adolescents: Prevalence and risk factors. Computers in Human Behavior2013; 29(5): 1987-96.

[53] Meerkerk GJ, Van Den Eijnden RJ, Vermulst AA, Garretsen HFL. The Compulsive Internet Use Scale (CIUS): Some psychometric properties. CyberPsychology \& Behavior2009 Feb; 12(1): 1-6.

[54] Thatcher A, Wretschko G, Fridjhon P. Online flow experiences, problematic Internet use and Internet procrastination. Computers in Human Behavior2008 Sep; 24(5): 2236-54

[55] Demetrovics Z, Szeredi B, Rozsa S. The three-factor model of Internet addiction: The development of the Problematic Internet Use Questionnaire. Behavior Research Methods2008 May; 40(2): 563-74.

[56] Cuhadar C. Exploration of problematic Internet use and social interaction anxiety among Turkish pre-service teachers. Computers \& Education2012 Sep; 59(2): 173-81.

[57] Huang Z, Wang M, Qian M, Zhong J, Tao R. Chinese Internet Addiction Inventory: Developing a measure of problematic Internet use for Chinese college students. Cyberpsychology \& Behavior2007 Dec; 10(6): 805-11.

[58] Bergmark KH, Bergmark A, Findahl O. Extensive Internet involvement: Addiction or emerging lifestyle? International Journal of Environmental Research and Public Health2011 Dec; 8(12): 4488-501.

[59] Beutel ME, Braehler E, Glaesmer H, Kuss DJ, Woelfling K, Mueller KW. Regular and problematic leisure-time Internet use in the community: Results from a German population-based survey. Cyberpsychology, Behavior and Social Networking2011 May; 14(5): 291-6.

[60] Kuss DJ, Griffiths MD, Binder JF. Internet addiction in students: Prevalence and risk factors. Computers in Human Behavior2013; 29(3): 959-66.

[61] Ak S, Koruklu N, Yilmaz Y. A study on Turkish adolescent's Internet use: Possible predictors of Internet addiction.
CyberPsychology, Behavior and Social Networking2013 2013-Mar; 16(3): 205-9.

[62] Poli R, Agrimi E. Internet addiction disorder: Prevalence in an Italian student population. Nordic Journal of Psychiatry2012 Feb; 66(1): 55-9.

[63] Cao H, Sun Y, Wan Y, Hao J, Tao F. Problematic Internet use in Chinese adolescents and its relation to psychosomatic symptoms and life satisfaction. BMC Public Health2011 Oct 14; 11.

[64] Wang H, Zhou X, Lu C, Wu J, Deng X, Hong L. Problematic Internet use in high school students in Guangdong Province, China. Plos One2011 May 6; 6(5).

[65] Lam LT, Peng ZW, Mai JC, Jing J. Factors associated with Internet addiction among adolescents. Cyberpsychology \& Behavior2009; 12(5): 551-5.

[66] Choi K, Son H, Park M, Han J, Kim K, Lee B, Gwak H. Internet overuse and excessive daytime sleepiness in adolescents. Psychiatry and Clinical Neurosciences2009 2009; 63(4): 455-62.

[67] Ha JH, Yoo HJ, Cho IH, Chin B, Shin D, Kim JH. Psychiatric comorbidity assessed in Korean children and adolescents who screen positive for Internet addiction. Journal of Clinical Psychiatry2006 May; 67(5): 821-6.

[68] Guo J, Chen L, Wang X, Liu Y, Chui CHK, He H, Qu Z, Tian D. The relationship between Internet addiction and depression among migrant children and left-behind children in China. Cyberpsychology, Behavior and Social Networking2012 Nov; 15(11): 585-90

[69] Siomos K, Floros G, Fisoun V, Evaggelia D, Farkonas N, Sergentani E, Lamprou M, Geroukalis D. Evolution of Internet addiction in Greek adolescent students over a two-year period: the impact of parental bonding. European child \& adolescent psychiatry2012 2012-Apr; 21(4): 211-9.

[70] Siomos KE, Dafouli ED, Braimiotis DA, Mouzas OD, Angelopoulos NV. Internet addiction among Greek adolescent students. Cyberpsychology \& Behavior2008 Dec; 11(6): 653-7.

[71] Fisoun V, Floros G, Geroukalis D, Ioannidi N, Farkonas N, Sergentani E, Angelopoulos N, Siomos K. Internet addiction in the island of Hippocrates: The associations between internet abuse and adolescent off-line behaviours. Child and Adolescent Mental Health2012 Feb; 17(1): 37-44.

[72] Fisoun V, Floros G, Siomos K, Geroukalis D, Navridis K. Internet addiction as an important predictor in early detection of adolescent drug use experience: Implications for research and practice. Journal of Addiction Medicine2012 Mar; 6(1): 77-84.

[73] Shek DTL, Yu L. Internet addiction phenomenon in early adolescents in Hong Kong. The Scientific World Journal2012 2012; 2012: 104304-.

[74] Gong J, Chen X, Zeng J, Li F, Zhou D, Wang Z. Adolescent addictive internet use and drug abuse in Wuhan, China. Addiction Research \& Theory2009 2009; 17(3): 291-305.

[75] Lin C-H, Lin S-L, Wu C-P. The effects of parental monitoring and leisure boredom on adolescents' Internet addiction. Adolescence2009 Win; 44(176): 993-1004.

[76] Johansson A, Gotestam KG. Internet addiction: Characteristics of a questionnaire and prevalence in Norwegian youth (12-18 years). Scandinavian Journal of Psychology2004 Jul; 45(3): 223-9.

[77] Kaltiala-Heino R, Lintonen T, Rimpela A. Internet addiction? Potentially problematic use of the Internet in a population of 12-18 year-old adolescents. Addiction Research \& Theory2004 Feb; 12(1): 89-96.

[78] Wang L, Luo J, Bai Y, Kong J, Gao W, Sun X. Internet addiction of adolescents in China: Prevalence, predictors, and association with well-being. Addiction Research \& Theory2013 2013; 21(1): 62-9.

[79] Ko CH, Yen JY, Chen CS, Yeh YC, Yen CF. Predictive values of psychiatric symptoms for Internet addiction in adolescents: A 2year prospective study. Archives of Pediatrics \& Adolescent Medicine2009 Oct; 163(10): 937-43.

[80] Ko CH, Yen JY, Liu SC, Huang CF, Yen CF. The associations between aggressive behaviors and Internet addiction and online activities in adolescents. Journal of Adolescent Health2009 Jun; 44(6): 598-605.

[81] Yen CF, Ko CH, Yen JY, Chang YP, Cheng CP. Multi-dimensional discriminative factors for Internet addiction among adolescents regarding gender and age. Psychiatry and Clinical Neurosciences2009; 63(3): 357-64.

[82] Ko C-H, Yen J-Y, Yen C-F, Chen C-S, Weng C-C, Chen C-C. The association between Internet addiction and problematic alcohol use 
in adolescents: The Problem Behavior Model. CyberPsychology \& Behavior2008 Oct; 11(5): 571-6.

[83] Ko CH, Yen JY, Yen CF, Chen CS, Wang SY. The association between Internet addiction and belief of frustration intolerance: The gender difference. Cyberpsychology \& Behavior2008 Aug; 11(3): 273-8.

[84] Yen J-Y, Ko C-H, Yen C-F, Chen S-H, Chung W-L, Chen C-C. Psychiatric symptoms in adolescents with Internet addiction: Comparison with substance use. Psychiatry and Clinical Neurosciences2008 Feb; 62(1): 9-16.

[85] Yen JY, Yen CF, Chen CC, Chen SH, Ko CH. Family factors of Internet addiction and substance use experience in Taiwanese adolescents. CyberPsychology \& Behavior2007 Jun; 10(3): 323-9.

[86] Ko CH, Yen JY, Chen CC, Chen SH, Wu K, Yen CF. Tridimensional personality of adolescents with Internet addiction and substance use experience. Canadian Journal of Psychiatry2006; 51(14): 887-94.

[87] Yen JY, Ko CH, Yen CF, Wu HY, Yang MJ. The comorbid psychiatric symptoms of Internet addiction: Attention deficit and hyperactivity disorder (ADHD), depression, social phobia, and hostility. Journal of Adolescent Health2007 Jul; 41(1): 93-8.

[88] Kim DI, Jeng YJ, Lee EA, Kim DM, Joe YM. Development of Internet Addiction Proneness Scale - Short Form (KS-Scale). Korean Journal of Counseling2008; 9: 1703-22.

[89] Barke A, Nyenhuis N, Kroener-Herwig B. The German Version of the Internet Addiction Test: A Validation Study. Cyberpsychology Behavior and Social Networking2012 Oct; 15(10): 534-42.

[90] Canan F, Ataoglu A, Ozcetin A, Icmeli C. The association between Internet addiction and dissociation among Turkish college students. Comprehensive Psychiatry2012 Jul; 53(5): 422-6.

[91] Yates TM, Gregor MA, Haviland MG. Child maltreatment, alexithymia, and problematic Internet use in young adulthood. Cyberpsychology, Behavior and Social Networking2012 Apr; 15(4): 219-25.

[92] Morrison CM, Gore $\mathrm{H}$. The relationship between excessive Internet use and depression: A questionnaire-based study of 1,319 young people and adults. Psychopathology2010; 43(2): 121-6.

[93] Whang LSM, Lee S, Chang G. Internet over-users' psychological profiles: A behavior sampling analysis on Internet addiction. Cyberpsychology \& Behavior2003 Apr; 6(2): 143-50.

[94] Ni X, Yan H, Chen S, Liu Z. Factors influencing Internet addiction in a sample of freshmen university students in China. Cyberpsychology \& Behavior2009 Jun; 12(3): 327-30.

[95] Kheirkhah F, Juibary AG, Gouran A. Internet addiction, prevalence and epidemiological features in Mazandaran Province, Northern Iran. Iranian Red Crescent Medical Journal2010 Mar; 12(2): 133-7.

[96] Bakken IJ, Wenzel HG, Gotestam KG, Johansson A, Oren A. Internet addiction among Norwegian adults: A stratified probability sample study. Scandinavian Journal of Psychology2009 Apr; 50(2): 121-7.

[97] Huang RL, Lu Z, Liu JJ, You YM, Pan ZQ, Wei Z, He Q, Wang ZZ. Features and predictors of problematic internet use in Chinese college students. Behaviour \& Information Technology2009 2009; 28(5): 485-90.

[98] Lin M-P, Ko H-C, Wu JY-W. Prevalence and psychosocial risk factors associated with Internet addiction in a nationally representative sample of college students in Taiwan. Cyberpsychology, Behavior and Social Networking2011 Dec; 14(12): 741-6.

[99] Yen JY, Yen CF, Wu HY, Huang CJ, Ko CH. Hostility in the real world and online: The effect of Internet addiction, depression, and online activity. Cyberpsychology, Behavior \& Social Networking2011; 14(11): 649-55.

[100] Tsai HF, Cheng SH, Yeh TL, Shih CC, Chen KC, Yang YC. The risk factors of Internet addiction - A survey of university freshmen. Psychiatry Research2009; 167(3): 294-9.

[101] Yen J-Y, Yen C-F, Chen C-S, Tang T-C, Ko C-H. The association between adult ADHD symptoms and Internet addiction among college students: The gender difference. Cyberpsychology \& Behavior2009 Apr; 12(2): 187-91.

[102] Yen JY, Ko CH, Yen CF, Chen CS, Chen CC. The association between harmful alcohol use and Internet addiction among college students: Comparison of personality. Psychiatry and Clinical Neurosciences2009; 63(2): 218-24.

[103] Lin M-P, Ko H-C, Wu JY-W. The role of positive/negative outcome expectancy and refusal self-efficacy of Internet use on
Internet addiction among college students in Taiwan. Cyberpsychology \& Behavior2008 Aug; 11(4): 451-7.

[104] Yeh Y-C, Ko H-C, Wu JY-W, Cheng C-P. Gender differences in relationships of actual and virtual social support to Internet addiction mediated through depressive symptoms among college students in Taiwan. Cyberpsychology \& Behavior2008 2008-Aug; 11(4): 485-7.

[105] American Psychiatric Association. Diagnostic and Statistical Manual for Mental Disorders IV, Text-Revision. Washington, D. C.: American Psychiatric Association; 2000.

[106] Caplan SE. Problematic Internet use and psychosocial well-being: Development of a theory-based cognitive-behavioral measurement instrument. Computers in Human Behavior2002 Sep; 18(5): 55375.

[107] Davis RA. A cognitive-behavioral model of pathological Internet use. Computers in Human Behavior2001 Mar; 17(2): 187-95.

[108] Caplan SE. Theory and measurement of generalized problematic Internet use: A two-step approach. Computers in Human Behavior2010; 26(5): 1089-97.

[109] Beranuy M, Oberst, U., Carbonell, X., \& Chamarro, A. Problematic Internet and mobile phone use and clinical symptoms in college students: The role of emotional intelligence. Computers in Human Behavior2009; 25(5): 1182-7.

[110] Grant JE, Levine L, Kim D, Potenza MN. Impulse control disorders in adult psychiatric inpatients. American Journal of Psychiatry2005 Nov; 162(11): 2184-6.

[111] Wölfling K, Müller K, Beutel M. Diagnostic measures: Scale for the Assessment of Internet and Computer Game Addiction (AICAS). In: Mücken D, Teske A, Rehbein F, te Wildt B, editors. Prevention, diagnostics, and therapy of computer game addiction. Lengerich: Pabst Science; 2010. p. 212-5.

[112] World Health Organization. ICD 10: The ICD-10 classification of mental and behavioral disorders: Clinical descriptions and diagnostic guidelines. Geneva, Switzerland: World Health Organization; 1992.

[113] Thatcher A, Goolam S. Development and psychometric properties of the Problematic Internet Use Questionnaire. South African Journal of Psychology2005; 35: 793-809.

[114] Lesieur HR, Blume SB. The South Oaks Gambling Screen (SOGS): A new instrument for the identification of pathological gamblers. American Journal of Psychiatry 1987; 144: 1184-8.

[115] Nyikos E, Szeredi B, Demetrovics Z. Egy új viselkedéses addikció: $\mathrm{Az}$ Internethasználat személyiségpszichológiai korrelátumai [A new behavioral addiction: The personality psychological correlates of Internet use]. Pszichoterápia2001; 10: 168-82.

[116] Ceyhan E, Ceyhan AA, Gürcan A. The validity and reliability of the Problematic Internet Usage Scale. Educational Sciences: Theory \& Practice2007; 7(1): 411-6.

[117] Beard KW, Wolf EM. Modification in the proposed diagnostic criteria for internet addiction. Cyberpsychology \& Behavior2001 Jun; 4(3): 377-83.

[118] Tao FB, Hu CL, Sun YH, Hao JH. The development and application of multidimensional sub-health questionnaire of adolescents (MSQA). Chinese Journal of Disorder Control and Prevention2008; 12: 309-14.

[119] Tian LL, Liu W. Test of the Chinese version of multidimensional students' life satisfaction scale. Chinese Mental Health Journal2005; 19: 301-3.

[120] Young KS. Internet addiction test (IAT). 2009 [10.04.2013]; Available from: http: //www.netaddiction.com/index.php?option=com_bfquiz\&view=one page \& catid $=46 \&$ Itemid $=106$.

[121] Zung W. A self-rating depression scale. Archives of General Psychiatry 1965; 12: 63-70.

[122] Kim HS. Internet addiction. Seoul: Nanum Press; 2000

[123] Johns MW. A new method for measuring daytime sleepiness: The Epworth Sleepiness Scale. Sleep1991; 14: 540-5.

[124] Kim K, Ryu E, Chon MY, Yeun EJ, Choi SY, Seo JS, Nam BW. Internet addiction in Korean adolescents and its relation to depression and suicidal ideation: A questionnaire survey. International Journal of Nursing Studies2006 Feb; 43(2): 185-92.

[125] Korean Neuropsychiatry Association. 1998 Korean depression screening day. Seoul: Korean Neuropsychiatry Association; 1999.

[126] Reynolds WM. Suicidal Ideation Questionnaire: Professional manual. Lutz, FL: Psychological Assessment Resources; 1988. 
[127] Kovacs M. The Children's Depression Inventory (CDI). North Tonawanda, NY: Multi-Health Systems; 2004.

[128] Siomos KE, Floros G, Mouzas OD, Angelopoulos NV. Validation of Adolescent Computer Addiction Test at a Greek sample. Psychiatriki2009; 20(3): 222-32.

[129] Parker GJM. The parental bonding instrument. Social Psychiatry and Psychiatric Epidemiology1990; 25(6): 281-2.

[130] Gossop M, Eysenck S. A further investigation into the personality of drug addicts in treatment. British Journal of Addiction1980; 75(3): 305-11.

[131] Young K. Internet addiction: Evaluation and treatment. Student British Medical Journal1999; 7: 351-2.

[132] Chappel JN, Veach TL, Krug RS. The substance abuse attitude survey: An instrument for measuring attitudes. Journal of Studies on Alcohol and Drugs 1985; 46(1): 48-52.

[133] Patterson GR, Stouthamer-Loeber M. The correlation of family management practices and delinquency. Child Development1984; 55: 1299-307.

[134] Iso-Ahola SE, Weissinger E. Perceptions of boredom in leisure: Conceptualization, reliability and validity of the leisure boredom scale. Journal of Leisure Research1990; 22(1): 1-17.

[135] Center for On-Line Addiction. The Internet Addiction Test. 2001 [12.04.2013]; Available from: http: //www.netaddiction.com/index.php?option=com_bfquiz\&view=one page \& catid $=46 \&$ Itemid $=106$.

[136] Kuang X, Cao Y, Dai X. Meta-analysis of Internet addiction scales' reliability and validity. Chinese Journal of Clinical Psychology and Psychotherapy2011; 19: 192-4.

[137] Chien CP, Cheng TA. Depression in Taiwan: Epidemiological survey utilizing CES-D. Seishin Shinkeigaku Zasshi - Psychiatria et Neurologia Japonica 1985; 87(5): 335-8.

[138] Rosenberg M, Schooler C, Schoenbach C. Self-esteem and adolescent problems: Modeling reciprocal effects. American Sociological Review1989; 54: 1004-18.

[139] Zhang F, Gao W. Development and validation of Chinese Adolescent Lifestyles Scale. Chinese Mental Health Journal for Specialists in Pediatric Nursing2010; 24: 515-9.

[140] Wolraich ML, Lambert W, Doffing MA, Bickman L, Simmons T, Worley K. Psychometric properties of the Vanderbilt ADHD Diagnostic Parent Rating Scale in a referred population. Journal of Pediatric Psychology2003; 28(8): 559-67.

[141] Leary MR. A brief version of the Fear of Negative Evaluation Scale. Personality and Social Psychology Bulletin1983; 9(3): 3715.

[142] Lin TK, Weng CY, Wang WC, Chen CC, Lin IM, Lin CL. Hostility trait and vascular dilatory functions in healthy Taiwanese. Journal of Behavioral Medicine2008; 31(6): 517-24.

[143] McConville DW, Cornell DG. Aggressive attitudes predict aggressive behavior in middle school students. Journal of Emotional and Behavioral Disorders2003; 11: 179-87.

[144] Smilkstein G. The family APGAR: A proposal for a family function test and its use by physicians. Journal of Family Practice 1978; 6: 1231-9.

[145] Rosenberg M. Conceiving the self. Malabar, FL: Krieger; 1986

[146] Radloff LS. The CES-D Scale: A self-report depression scale for research in the general population. Applied Psychological Measurement 1977; 1: 385-401.

[147] Yen CF, Yang YS, Chong MY. Correlates of methamphetamine use for Taiwanese adolescents. Psychiatry and Clinical Neurosciences2006; 60: 160-7.

[148] Yen CF, Shieh BL. Suicidal ideation and correlates in adolescent methamphetamine users. The Journal of Nervous and Mental Disease2006; 193: 444-9.

[149] Rosenberg M. Society and adolescent self-image. New Jersey: Princeton University Press; 1965.

[150] Knight JR, Shrier LA, Bravender TD, Farrell M, Vander Bilt J, Shaffer HJ. A new brief screen for adolescent substance abuse. Archives of Pediatrics and Adolescent Medicine 1999; 153(6): 5916.

[151] Gray JA. The neuropsychology of temperament. In: Strelau J, Angleitner A, editors. Explorations in temperament: International perspectives on theory and measurement. New York: Plenum Press; 1991. p. 105-28.

[152] Zullig KJ, Huebner ES, Gilman R, Patton JM, Murray KA. Validation of the brief multidimensional students' life satisfaction scale among college students. . American Journal of Health Behavior2005; 29(3): 206-14.

[153] Harrington N. The Frustration Discomfort Scale: Development and psychometric properties. Clinical Psychology and Psychotherapy2005; 12: 374-87.

[154] Derogatis LR, Melisaratos N. The Brief Symptom Inventory: An introductory report. Psychological Medicine1983; 13: 595-605.

[155] Yen CF, Yang YH, Ko CH, Yen JY. Substance initiation sequences among Taiwanese adolescents using methamphetamine. Psychiatry and Clinical Neurosciences2005; 59: 683-9.

[156] Kuo PH, Yang HJ, Soong WT, Chen WJ. Substance use among adolescents in Taiwan: Associated personality traits, incompetence, and behavioral/emotional problems. Drug and Alcohol Dependence2002; 67: 27-39.

[157] Yen J-Y, Ko C-H, Yen C-F, Wu H-Y, Yang M-J. The comorbid psychiatric symptoms of Internet addiction: Attention deficit and hyperactivity disorder (ADHD), depression, social phobia, and hostility. Journal of Adolescent Health2007 Jul; 41(1): 93-8.

[158] Connor KM, Davidson JRT, Churchill LE, Sherwood A, Weisler RH. Psychometric properties of the Social Phobia Inventory (SPIN): New self-rating scale. The British Journal of Psychiatry2000; 176(4): 379-86.

[159] Lin IM, Weng CY. Relationship between hostility pattern and psychophysiological disorders: Cases of coronary artery disease and headache. Chinese Journal of Psychology2002; 44: 211-26.

[160] Vermulst AA, Gerris JRM. QBF: Quick Big Five Persoonlijkheidstest Handleiding [Quick Big Five Personality Test Manual]. Leeuwarden, NL: LDC Publications; 2005.

[161] Russell D, Peplau LA, Cutrona CE. The revised UCLA Loneliness Scale: Concurrent and discriminant validity evidence. Journal of Personality and Social Psychology 1980; 39: 472-80.

[162] Rosenberg M. Society and the Adolescent Self-Image. Middletown: Wesleyan University Press; 1989.

[163] Kandel DB, Davies M. Adult sequelae of adolescent depressive symptoms. Archives of General Psychiatry 1986; 43: 255-62.

[164] Pereda N, Forns M, Peró M. Dimensional structure of the Brief Symptom Inventory with Spanish college students. Psicothema2007; 19(4): 634-9.

[165] Chico E, Tous JM, Lorenzo-Seva U, Vigil-Colet A. Spanish adaptation of Dickman's Impulsivity Inventory: Its relationship to Eysenck's Personality Questionnaire. Personality and Individual Differences2003; 35(8): 1883-92.

[166] Dickman SJ. Functional and dysfunctional impulsivity: Personality and cognitive correlates. Journal of Personality and Social Psychology1990; 58(1): 95-102.

[167] DRM Study Group. DRM 52 scale of internet use in adolescents. In: Shanghai Youth Federation HKoYG, and Singapore People's Association Youth Movement, editor. Adolescent internet addiction: Recognition and intervention. Shanghai: East China University of Science and Technology Press; 2006. p. 34-40.

[168] Beranuy M, Chamarro A, Graner C, Carbonell X. Validación de dos escalas breves para evaluar la adicción a Internet y el abuso de móvil. Psicothema2009; 21(3): 480-5.

[169] Davis RA. Problematic internet use: Structure of the construct and association with personality, stress, and coping. Dissertation Abstracts International Section B2004; 65: 472.

[170] Nichols LA, Nicki R. Development of a psychometrically soun Internet addiction scale: A preliminary step. Psychology of Addictive Behaviors2004 Dec; 18(4): 381-4.

[171] Bernstein EM, Putnam FW. Development, reliability, and validity of a dissociation scale. The Journal of Nervous and Mental Disease1986; 174(2): 727-35.

[172] Sanders B, Becker-Lausen E. The measurement of psychological maltreatment: early data on the child abuse and trauma scale. Child Abuse and Neglect 1995; 19(3): 315-23.

[173] Bagby RM, Parker JDA, Taylor GJ. The twenty-item Toronto Alexithymia Scale: I. Item selection and cross-validation of the factor structure. Journal of Psychosomatic Research1994; 38(1): 23-32.

[174] Broadhead WE, Gehlbach SH, de Gruy FV, Kaplan BH. The DukeUNC Functional Social Support Questionnaire: Measurement of social support in family medicine patients. Medical Care1988; 26(7): 709-23.

[175] Derogatis LR. SCL-90-R: Administration, scoring, and procedures manual II for the revised version. Towson, MD: Clinical Psychometric Research; 1983. 
[176] Beck AT, Ward CH, Mendelson M, Mock J, Erbaugh J. An inventory for measuring depression. Archives of General Psychiatry 1961; 4(6): 561-71.

[177] Zung WW. A rating instrument for anxiety disorders. Psychosomatics1971; 12: 371-9.

[178] Kitamura T, Sugawara M, Shima S, Toda MA. Temporal variation of validity of self-rating questionnaires: Improved validity of repeated use of Zung's selfrating depression scale among women during the perinatal period. Journal of Psychosomatic Obstetrics \& Gynaecology1999; 20(2): 112-7.

[179] Ko CH, Yen JY, Chen SH, Yang MJ, Lin HC, Yen CF. Proposed diagnostic criteria and the screening and diagnosing tool of Internet addiction in college students. Comprehensive Psychiatry2009 JulAug; 50(4): 378-84.

[180] Chiu SH, Ko HC, Wu JY. Depression moderated the effect of exposure to suicide news on suicidality among college students in Taiwan. Suicide and Life-Threatening Behavior2007; 37(5): 58592.

[181] Li CH, Ko HC, Weng LJ, Liau L-C, Lu R-B. The development of an impulsiveness scale: Psychometric properties and relation to antisocial personality disorder. Chinese Journal of Psychology2002; 44(1): 109-19.

[182] Bartholomew K, Horowitz LM. Attachment styles among young adults: A test of a four-category model. Journal of Personality and Social Psychology Bulletin1991; 61(2): 226-44.

[183] Chong MY, Wilkinson G. Validation of 30- and 12-item versions of the Chinese Health Questionnaire (CHQ) in patients admitted for general health screening. Psychological Medicine1989; 19: 495505 .

[184] Berkman LF, Glass T. Social integration, social networks, social support, and health. In: Berkman LF, Kawachi I, editors. Social Epidemiology. Oxford: Oxford University Press; 2000. p. 137-73.

[185] Eysenck HJ, Eysenck SBG. Manual of the Eysenck Personality Questionnaire. London: Hodder \& Stoughton; 1975.

[186] Kessler RC, Adler L, Ames M, Demler O, Faraone S, Hiripi E, Howes MJ, Jin R, Secnik K, Spencer T, Ustun TB, Walters EE. The World Health Organization Adult ADHD Self-Report Scale (ASRS): A short screening scale for use in the general population. Psychological Medicine2005; 35(2): 245-56.

[187] Babor T, Fuente J, Saunders J, Grant M. The Alcohol Use Disorders Identification Test: Guidelines for use in primary health care. Geneva: Division of Mental Health, World Health Organization; 1989

[188] Ko HC. The comparison of the dexamethasone suppression test, dysfunctional attitude style, and life stress between patients with endogenous and nonendogenous depression. Taipei: Institute of Psychology, National Taiwan University; 1989.

[189] Costa PT, McCrae RR. Revised NEO personality inventory (NEOPI-R) and the NEO Five-Factor inventory (NEO-FFI): Professional manual. Odessa, FL: Psychological Assessment Resources; 1992.

[190] Webster J, Trevino LK, Ryan L. The dimensionality and correlates of flow in human-computer interactions. Computers in Human Behavior 1993; 9: 411-29.

[191] Ceyhan E, Ceyhan AA, Gürcan A. Problemli Internet kullanımı ölçe gi'nin geçerlik ve güvenirlik çalısmaları. [The validity and reliability of the problematic Internet usage scale]. Kuram ve Uygulamada Egitim Bilimleri [Educational Sciences: Theory \& Practice]2007; 7(1): 387-416.

[192] Sübası G. Etkilesim kaygısı ölçeginin gelistirilmesi: geçerlik ve güvenirlik çalısmaları. [The development of interaction anxiousness scale: Studies on the validity and reliability of the scale]. Türk Psikolojik Danısma ve Rehberlik Dergisi [Journal of Turkish Psychological \& Guidance]2003; 2(19): 35-40.

[193] Michal M, Zwerenz R, Tschan R, Edinger J, Lichy M, Knebel A, Tuin I, Beutel M. Screening for depersonalization-derealization with two items of the Cambridge Depersonalization Scale. Psychotherapie, Psychosomatik, Medizinische Psychologie2010; 60(5): 175-9.

[194] Hinz A, Schwarz R. Anxiety and depression in the general population: Normal values in the Hospital Anxiety and Depression Scale. Psychotherapie, Psychosomatik, Medizinische Psychologie2001; 51: 193-200.

[195] Wang C-C, Chen C-F, Chen C-T. Exploring the different aspects of Internet leisure use by college students. Information Development2013 July 5, 2013.
[196] Xu Z, Yuan Y, editors. The impact of motivation and prevention factors on game addiction. Special Interest Group on HumanComputer Interaction 2008; 2008: Association for Information Systems.

[197] First MB. Clinical utility: A prerequisite for the adoption of dimensional approach in DSM. Journal of Abnormal Psychology2005 Nov; 114(4): 560-4.

[198] Kraemer HC. DSM categories and dimensions in clinical and research contexts. International Journal of Methods in Psychiatric Research2007 Jun; 16: S8-S15.

[199] Watson D. Rethinking the mood and anxiety disorders: A quantitative hierarchical model for DSM-V. Journal of Abnormal Psychology2005 Nov; 114(4): 522-36.

[200] Widiger TA, Samuel DB. Diagnostic categories or dimensions? A question for the Diagnostic and Statistical Manual of Mental Disorders - Fifth edition. Journal of Abnormal Psychology2005 Nov; 114(4): 494-504

[201] Helsper E, Gerber MM. The plausibility of cross-national comparisons of Internet use types. Information Society2012 2012; 28(2): 83-98.

[202] Griffiths MD. The use of online methodologies in data collection. International Journal of Mental Health and Addiction2010; 8(1): 820.

[203] Church AT, Lonner WJ. The cross-cultural perspective in the study of personality. Journal of Cross-Cultural Psychology1998; 29(1): 32-62.

[204] Frankfort-Nachmias C, Nachmias D. Research methods in the social sciences. 5 ed. London: St Martin's Press; 1996.

[205] Polaino A, Senra C. Measurement of depression - Comparison between self-reports and clinical assessments of depressed outpatients. Journal of Psychopathology and Behavioral Assessment1991 Dec; 13(4): 313-24.

[206] Cheshire K, Pilgrim D. A short introduction to clinical psychology. London: Sage; 2004.

[207] Ruben DH. Diagnosing alcoholism and its addictive patterns using self-report rating scales. Alcoholism Treatment Quarterly 1999; 17(3): 37-46.

[208] Jackson JL, O'Malley PG, Kroenke K. Clinical predictors of mental disorders among medical outpatients - Validation of the "S4" model. Psychosomatics1998 Sep-Oct; 39(5): 431-6.

[209] Waldeck TL, Miller LS. Gender and impulsivity differences in lici substance use. Journal of Substance Abuse Treatment1997; 9: 26975 .

[210] Billieux J, Rochat L, Ceschi G, Carré A, Offerlin-Meyer I, Defeldre A-C, Khazaal Y, Besche-Richard C, Van der Linden M. Validation of a short French version of the UPPS-P Impulsive Behaviour Scale. Comprehensive Psychiatry2012; 54(5): 609-15

[211] Kessler RC, Avenevoli S, Costello EJ, Georgiades K, Green JG, Gruber MJ, He J-p, Koretz D, McLaughlin KA, Petukhova M, Sampson NA, Zaslavsky AM, Merikangas KR. Prevalence, persistence, and sociodemographic correlates of DSM-IV disorders in the National Comorbidity Survey Replication Adolescent Supplement. Archives of General Psychiatry2012 Apr; 69(4): 37280

[212] Csikszentmihályi M. Flow: The psychology of optimal experience New York: HarperCollins; 1990

[213] Lombard M, Ditton T. At the heart of it all: The concept of presence. Journal of Computer-Mediated Communication1997; 3(2): 0-.

[214] Stavropoulos V, Alexandraki K, Motti-Stefanidi F. Flow and telepresence contributing to Internet abuse: Differences according to gender and age. Computers in Human Behavior2013; 29(5): 1941-8.

[215] Kuss DJ, Griffiths MD. Internet gaming addiction: A systematic review of empirical research. International Journal of Mental Health and Addiction2012; 10(2): 278-96

[216] Kuss DJ, Griffiths MD. Online social networking and addiction - A review of the psychological literature. International Journal of Environmental Research and Public Health2011 Sep; 8(9): 352852

[217] Lin SSJ, Tsai CC. Sensation seeking and internet dependence of Taiwanese high school adolescents. Computers in Human Behavior2002 Jul; 18(4): 411-26.

[218] Kuss DJ, Louws J, Wiers RWW. Online gaming addiction? Motives predict addictive play behavior in Massively Multiplayer 
Online Role-Playing Games Cyberpsychology, Behavior \& Social Networking2012; 15(9): 480-5.

[219] Billieux J, Van der Linden M, Achab S, Khazaal Y, Paraskevopoulos L, Zullino D, Thorens G. Why do you play World of Warcraft? An in-depth exploration of self-reported motivations to play online and in-game behaviours in the virtual world of Azeroth. Computers in Human Behavior2013; 29(1): 103-9.

[220] Cooper ML, Russell M, George WH. Coping, expectancies, and alcohol abuse: A test of social learning formulations. Journal of Abnormal Psychology 1988; 97(2): 218-30.

[221] Gentile DA, Choo H, Liau A, Sim T, Li DD, Fung D, Khoo A. Pathological video game use among youths: A two-year longitudinal study. Pediatrics2011; 127(2): E319-E29.

Received: July 15, 2013

Accepted: August 26, 2013
[222] Drake RE, McLaughlin P, Pepper B, Minkoff K. Dual diagnosis of major mental illness and substance disorder: An overview. New Directions for Mental Health Services 1991; 1991(50): 3-12.

[223] Regier DA, Farmer ME, Rae DS, Locke BZ, Keith SJ, Judd LL, Goodwin FK. Comorbidity of mental disorders with alcohol and other drug abuse. Results from the Epidemiologic Catchment Area (ECA) Study. JAMA1990; 264(19): 2511-8.

[224] Shaffer HJ, LaPlante DA, LaBrie RA, Kidman RC, Donato AN, Stanton MV. Toward a syndrome model of addiction: Multiple expressions, common etiology. Harvard Review of Psychiatry2004 Nov-Dec; 12(6): 367-74

[225] Kuss DJ, Griffiths MD. Online gaming addiction in children and adolescents: A review of empirical research. Journal of Behavioral Addictions2012; 1(1): 3-22. 